\title{
Taylor drop in a closed vertical pipe
}

\author{
D. Picchi ${ }^{1, \dagger}$, J. Suckale S,3,4 $^{2,3}$ I. Battiato ${ }^{5,} \dagger$ \\ ${ }^{1}$ Department of Mechanical and Industrial Engineering, Università degli Studi di Brescia, \\ Brescia 25123, Italy \\ ${ }^{2}$ Department of Geophysics, Stanford University, Stanford, CA 94305, USA \\ ${ }^{3}$ Institute of Computational and Mathematical Engineering, Stanford University, \\ Stanford, CA 94305, USA \\ ${ }^{4}$ Department of Civil and Environmental Engineering, Stanford University, Stanford, CA 94305, USA \\ ${ }^{5}$ Department of Energy Resources Engineering, Stanford University, Stanford, CA 94305, USA
}

(Received 30 December 2019; revised 6 June 2020; accepted 14 July 2020)

In this work, we study the ascent dynamics of a liquid Taylor drop formed from a lock-exchange configuration in a closed vertical pipe. We focus on the buoyancy-driven motion of an elongated drop surrounded by a denser fluid when viscous forces dominate over inertial and surface tension effects. While gaseous Taylor bubbles have been studied extensively, a liquid Taylor drop moving in a closed pipe is less well understood. We formulate an analytical model for estimating the ascent speed and drop thickness from first principles. First, we use a lubrication approximation to solve for the velocity profiles in the two fluids. Then, we analyse the mechanical energy balance of the whole system, including the effect of viscous dissipation, to understand how the ascent speed and drop thickness scale with the viscosity ratio. We show that a drop with density ratio $\mathcal{R}$ reaches a stationary state with a uniform dimensionless thickness of $\sqrt{2} / 2$ in the absence of dissipation and $\sqrt{2 \mathcal{R}} / 2$ in the dissipative regime. Through a comparison with existing experimental data, we demonstrate that our model correctly predicts the ascent speed of a Taylor drop if the material properties of the fluids and the geometry of the conduit are known. Our theoretical framework can be generalized to an isolated Taylor drop rising in a vertical pipe.

Key words: gravity currents, multiphase flow, lubrication theory

\section{Introduction}

The motion of elongated drops in vertical pipes arises in many engineering systems, such as buoyancy-driven displacements in wells and chemical reactors (Baird et al. 1992; Frigaard \& Scherzer 1998; Sahu \& Vanka 2011; Alba, Taghavi \& Frigaard 2014; Hasnain \& Alba 2017; Hasnain, Segura \& Alba 2017; Etrati \& Frigaard 2018; Mirzaeian \& Alba 2018; Amiri et al. 2019; Oladosu et al. 2019). In nature, it is important for understanding conduit

$\dagger$ Email addresses for correspondence: davide.picchi@unibs.it, ibattiat@stanford.edu 

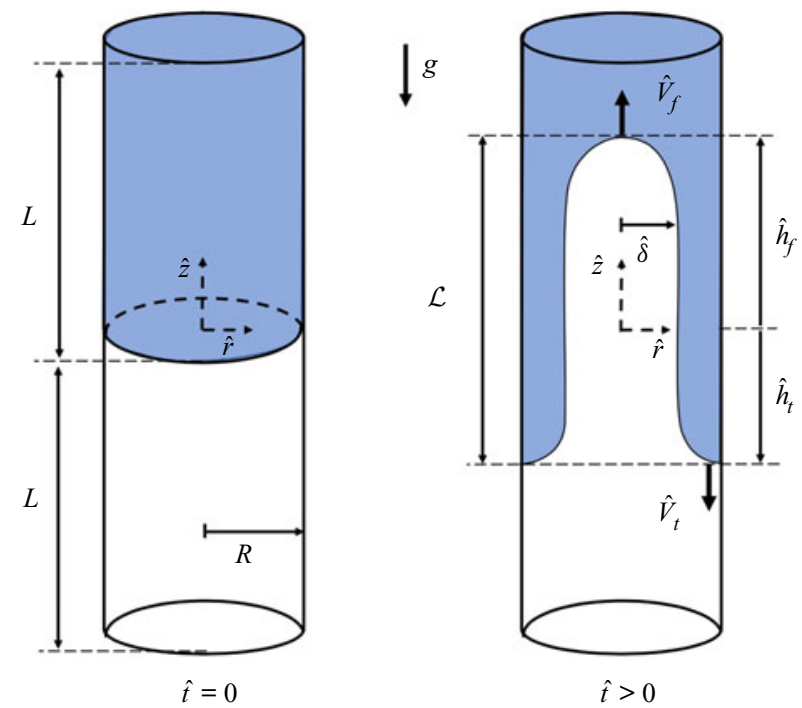

FIGURE 1. Sketch of the lock-exchange problem in a vertical pipe of length $2 L$ and radius $R$. At time $\hat{t}=0$, the top part of the pipe is filled with the heavier, descending fluid (dark blue) and the bottom part with the lighter, ascending fluid (white). When the lock is removed, a Taylor drop of length $\mathcal{L}$ and thickness $\hat{\delta}$ forms and the front ascends at speed $\hat{V}_{f}$ while the tail descends at speed $\hat{V}_{t}$.

flow in persistently degassing volcanoes (Francis, Oppenheimer \& Stevenson 1993; Kazahaya, Shinohara \& Saito 1994; Stevenson \& Blake 1998; Fowler \& Robinson 2018; Suckale et al. 2018). In all of these systems, gravity is the only driving force that generates the exchange flow of fluids with different densities.

A typical experimental mechanism that leads to the formation of elongated drops is the lock-exchange configuration. In this set-up, a heavier fluid is initially separated from a lighter fluid by a horizontal barrier. Retraction of this barrier at the onset of the experiment releases the heavier fluid into the lighter fluid (see figure 1). The light fluid takes a symmetrical bullet shape, commonly called a Taylor drop, while the heavy fluid sinks as a film along the domain walls. Although an analytic description for the ascent speed of the drop may be useful for many problems, including the experimental study of slug flows (Joseph \& Renardy 1992; Brauner 1998), the dynamics of an isolated Taylor drop moving in a closed environment has not yet been quantified in detail.

Previous research has focused primarily on understanding the ascent dynamics of a gaseous Taylor bubble (Dumitrescu 1943; Davies \& Taylor 1950; White \& Beardmore 1962; Brown 1965; Zukoski 1966; Viana et al. 2003). When capillary forces are negligible and inertial effects dominate over viscous forces, the Taylor bubble rises with a speed that is proportional to $\sqrt{g D}$. This result has been obtained theoretically by Dumitrescu (1943) and Davies \& Taylor (1950) using the potential flow theory for an ellipsoidal inviscid bubble and has later been confirmed by several experiments (White \& Beardmore 1962; Brown 1965; Zukoski 1966; Viana et al. 2003). When viscous forces dominate over inertial and surface tension effects, the ascent speed of a gaseous Taylor bubble is proportional to $\Delta \rho g D^{2} / \mu_{d}$, where $\mu_{d}$ is the viscosity of the liquid (Brown 1965; Wallis 1969). In contrast, when surface tension dominates over buoyancy, the ascent speed and film thickness are controlled by capillary forces (Bretherton 1961; Reinelt 1987; Batchelor 2000; Llewellin et al. 2012; Shukla et al. 2019). 
Most existing studies assume a density contrast of multiple orders of magnitude between the Taylor bubble and the surrounding fluid. In some natural systems, however, the two overturning fluids have similar densities, leading to the formation of Taylor drops rather than Taylor bubbles. One specific example where this flow configuration arises is the conduit of a persistently active volcano (e.g. Francis et al. 1993). Persistent activity is driven by degassing as evidenced by continual emissions of large quantities of gas, which are occasionally punctuated by the eruption of comparatively negligible quantities of magma (e.g. Vergniolle \& Mangan 2000). Originally dissolved in the magma, volatiles exsolve as the magma ascends, forming a large number of small bubbles. Most bubbles remain largely or partially entrained in the ambient flow due to the high magma viscosity. As a consequence, a Taylor drop of bubble-rich, buoyant magma can form and ascend to the surface where the bubbles escape, depriving the magma of its buoyancy and leaving it for recycling back to depth (e.g. Stevenson \& Blake 1998; Beckett et al. 2011; Kerswell 2011; Suckale et al. 2018). Since magma viscosity depends very sensitively on the dissolved volatile content and temperature, the viscosity between upwelling and downwelling magmas can vary by several orders of magnitude (Giordano, Russell \& Dingwell 2008).

Up to now, only the experiments by Stevenson \& Blake (1998) and Goldsmith \& Mason (1962) constrain the ascent dynamics of Taylor drops in a regime where viscous forces dominate over inertial and surface tension effects. While Goldsmith \& Mason (1962) investigated the motion of an isolated Taylor drop, Stevenson \& Blake (1998) focused primarily on vertical lock-exchange systems for a large range of viscosity contrasts. Stevenson \& Blake (1998) found that the non-dimensional rise speed of both isolated drops and elongated drops formed from a lock-exchange configuration saturates at a constant value when the viscosity of the surrounding fluid is approximately an order of magnitude higher than the viscosity of the drop. This finding has been confirmed by models (Suckale et al. 2018). Also, Kurimoto, Hayashi \& Tomiyama (2013) and Hayashi, Kurimoto \& Tomiyama (2011) measured the ascent speed of Taylor drops with and without the effect of surfactants when inertial forces are non-negligible. Direito, Campos \& Miranda (2016) showed numerically that the recirculation patterns inside a Taylor drop change significantly with the viscosity ratio.

The goal of this paper is to quantify the ascent dynamics of a Taylor drop confined in a vertical pipe when surface tension and inertia are negligible. We propose a theoretical framework that quantifies the interplay between buoyancy and viscous forces for an elongated Taylor drop. Our analytical model estimates the ascent speed and drop thickness from first principles and elucidates the effect of the viscosity ratio on the ascent dynamics of a Taylor drop. While our model is motivated primarily by the flow conditions arising in the conduits of persistently active volcanoes, as reflected in the non-dimensional regime we explore, it is generally applicable to Taylor drops in other fields of application.

We derive a lubrication model for a Taylor drop formed from a lock-exchange configuration and obtain the scaling of the ascent speed as a function of the drop thickness and the viscosity ratio $(\$ 2)$. The lubrication model solves for the velocity profiles in the drop and the surrounding fluid. To identify which configurations are stable, we analyse the energy balance of the whole system. We show that the drop obeys an ordinary differential equation for the drop thickness. The steady state is determined by the interplay of the potential energy and the viscous dissipation produced by the motion of the flowing fluids (§3). When the drop reaches its terminal ascent speed, its thickness is independent of the viscosity ratio in the non-dissipative regime $(\$ 4.1)$. 
Our theory provides a new theoretical framework that not only explains the lock-exchange experiments of Stevenson \& Blake (1998) (\$4.2), but that can also be generalized to the case of an isolated elongated drop released into a vertical pipe $(\S 4.3)$. Our theory agrees well with the measurements of Goldsmith \& Mason (1962) and provides new physical insights into the backflow patterns in Taylor drops in the presence of large variations in the viscosity contrast $(\S 4.4)$.

\section{Lubrication model}

\subsection{Governing equations}

In the lock-exchange configuration, the fluids are initially unstably stratified. An elongated Taylor drop forms after lock removal, as sketched in figure 1. Assuming immiscible flow of two Newtonian fluids, the governing equations are continuity and Navier-Stokes in both fluids

$$
\begin{gathered}
\nabla \cdot \hat{\boldsymbol{u}}_{i}=0, \\
\rho_{i}\left[\frac{\partial \hat{\boldsymbol{u}}_{i}}{\partial \hat{t}}+\left(\hat{\boldsymbol{u}}_{i} \cdot \nabla\right) \hat{\boldsymbol{u}}_{i}\right]=-\nabla \hat{p}+\mu_{i} \nabla^{2} \hat{\boldsymbol{u}}_{i}-\rho_{i} g \boldsymbol{e}_{g},
\end{gathered}
$$

where $\hat{p}$ is the pressure and $\hat{\boldsymbol{u}}=(\hat{v}, \hat{w}, \hat{u})$ is the velocity vector with $\hat{v}, \hat{w}$ and $\hat{u}$ representing the velocity components in the $r, \theta$ and $z$ directions, respectively. The subscripts, $i=$ $d, a$, refer to the descending and ascending fluid, respectively. The dynamic viscosity and density of the fluids are $\mu_{i}$ and $\rho_{i}$. The acceleration due to gravity is denoted by $g$ and acts in the direction of unit vector $\boldsymbol{e}_{g}$. The boundary conditions are no-slip and no-penetration at the pipe wall and continuity of velocity and shear stresses at the fluid-fluid interface. In addition, the system satisfies the exchange-flow condition, which requires that the net volumetric flux is zero in each cross-section $\hat{A}$, i.e.

$$
\int_{\hat{A}_{d}} \hat{\boldsymbol{u}}_{d} \cdot \boldsymbol{n} \mathrm{d} \hat{S}+\int_{\hat{A}_{a}} \hat{\boldsymbol{u}}_{a} \cdot \boldsymbol{n} \mathrm{d} \hat{S}=0,
$$

where $\boldsymbol{n}$ is the vector normal to the pipe cross-section. The dimensional parameters governing the flow are listed in table 1.

\subsection{Dimensionless formulation and lubrication approximation}

In this section, we derive a lubrication model for a Taylor drop of length $\mathcal{L}$ and thickness $\hat{\delta}$. We assume that the drop is sufficiently long such that the fluid-fluid interface is approximately parallel to the vertical direction, $\hat{z}$ in figure 1 . We can thus neglect the velocity component in the azimuthal direction, $\hat{w}_{i}=0$, which leaves $\hat{\boldsymbol{u}}_{i}=\left(\hat{v}_{i}, 0, \hat{u}_{i}\right)$. The two relevant length scales in the vertical and radial directions are the length of the drop, $\mathcal{L}$, and the pipe radius $R$. Based on these, we define the scale parameter,

$$
\varepsilon=\frac{R}{\mathcal{L}}
$$

The identification of the velocity scale is not trivial since the volumetric flux cannot be predicted a priori. The spreading of the drop in the axial direction is controlled by a balance between buoyancy and viscous stress. Buoyancy is the driving force and it scales as $\Delta \rho g R$, where $\Delta \rho=\rho_{d}-\rho_{a}$ is the density difference between the ascending and the 
Definition

Pipe radius

Gravitational acceleration

Drop length

Half of pipe length

Light fluid density

Heavy fluid density

Light fluid viscosity

Heavy fluid viscosity

Surface tension

Density difference

Velocity scale
Parameter

$R$

$g$

$\mathcal{L}$

$L$

$\rho_{a}$

$\rho_{d}$

$\mu_{a}$

$\mu_{d}$

$\sigma$

$\Delta \rho=\left(\rho_{d}-\rho_{a}\right)$

$U=\Delta \rho g R^{2} / \mu_{d}$
Definition

Drop thickness

Ascent speed

Descent speed

Front location

Tail location

Cross-sectional area

Light fluid cross-section

Heavy fluid cross-section

Kinetic energy

Potential energy

Viscous dissipation
Variable

$\hat{\delta}$
$\hat{V}_{f}$
$\hat{V}_{t}$
$\hat{h}_{f}$
$\hat{h}_{t}$
$\hat{A}=\pi R^{2}$
$\hat{A}_{a}=\pi \hat{\delta}^{2}$
$\hat{A}_{d}=\pi\left(R^{2}-\hat{\delta}^{2}\right)$
$\hat{\mathcal{E}}$
$\hat{\mathcal{P}}$
$\hat{\Phi}$

TABLE 1. List of the dimensional input parameters and variables of the problem.

descending fluid. The stress at the wall dissipates the energy and it scales as $\mu_{d} U / R$. By matching the two contributions, we obtain the scale for the axial velocity component

$$
\Delta \rho g R \sim \frac{\mu_{d} U}{R} \Rightarrow U=\frac{\Delta \rho g R^{2}}{\mu_{d}} .
$$

Using the continuity equations, we infer the characteristic scale of the radial component of the velocity as $\varepsilon U$.

We then normalize (2.1) by introducing the following dimensionless variables:

$$
z=\frac{\hat{z}}{\mathcal{L}}, \quad r=\frac{\hat{r}}{R}, \quad t=\frac{\hat{t}}{\mathcal{L} / U}, \quad u_{i}=\frac{\hat{u}_{i}}{U}, \quad v_{i}=\frac{\hat{v}_{i}}{\varepsilon U}, \quad p_{i}=\frac{\hat{p}_{i}}{\mu_{d} U \mathcal{L} / R^{2}} .
$$

We obtain two momentum equations in the axial and radial directions for the descending fluid

$$
\begin{gathered}
\varepsilon A r \frac{\mathrm{D} u_{d}}{\mathrm{D} t}=-\frac{\partial p_{d}}{\partial z}+\varepsilon \frac{\partial^{2} u_{d}}{\partial z^{2}}+\frac{1}{r} \frac{\partial}{\partial r}\left(r \frac{\partial u_{d}}{\partial r}\right)-\frac{1}{1-\mathcal{R}}, \\
\varepsilon^{2} A r \frac{\mathrm{D} v_{d}}{\mathrm{D} t}=-\frac{1}{\varepsilon} \frac{\partial p_{d}}{\partial r}+\varepsilon^{3} \frac{\partial^{2} v_{d}}{\partial z^{2}}+\varepsilon\left[\frac{1}{r} \frac{\partial}{\partial r}\left(r \frac{\partial v_{d}}{\partial r}\right)-\frac{v_{d}}{r^{2}}\right],
\end{gathered}
$$

and for the ascending fluid

$$
\begin{aligned}
\varepsilon \mathcal{R} A r \frac{\mathrm{D} u_{a}}{\mathrm{D} t} & =-\frac{\partial p_{a}}{\partial z}+\frac{\varepsilon}{M} \frac{\partial^{2} u_{a}}{\partial z^{2}}+\frac{1}{M r} \frac{\partial}{\partial r}\left(r \frac{\partial u_{a}}{\partial r}\right)-\frac{\mathcal{R}}{1-\mathcal{R}}, \\
\varepsilon^{2} \mathcal{R} A r \frac{\mathrm{D} v_{a}}{\mathrm{D} t} & =-\frac{1}{\varepsilon} \frac{\partial p_{a}}{\partial r}+\frac{\varepsilon^{3}}{M} \frac{\partial^{2} v_{a}}{\partial z^{2}}+\frac{\varepsilon}{M}\left[\frac{1}{r} \frac{\partial}{\partial r}\left(r \frac{\partial v_{a}}{\partial r}\right)-\frac{v_{a}}{r^{2}}\right]
\end{aligned}
$$

where

$$
\frac{\mathrm{D}}{\mathrm{D} t}=\frac{\partial}{\partial t}+v_{i} \frac{\partial}{\partial r}+u_{i} \frac{\partial}{\partial z}
$$


Definition

Scale parameter

Archimedes number

Density ratio

Viscosity ratio

Bond number

Richardson number
Parameter

$$
\begin{gathered}
\varepsilon=R / \mathcal{L} \\
A r=\rho_{d} \Delta \rho g R^{3} / \mu_{d}^{2} \\
\mathcal{R}=\rho_{a} / \rho_{d} \\
M=\mu_{d} / \mu_{a} \\
B o=\Delta \rho g R^{2} / \sigma \\
R i=g \mathcal{L} / U^{2}
\end{gathered}
$$

Definition

Drop thickness

Ascent speed

Descent speed

Front location

Tail location

Cross-sectional area

Light fluid cross-section

Heavy fluid cross-section

Kinetic energy

Potential energy

Viscous dissipation
Variable

$\delta=\hat{\delta} / R$

$V_{f}=\hat{V}_{f} / U$

$V_{t}=\hat{V}_{t} / U$

$h_{f}=\hat{h}_{f} / \mathcal{L}$

$h_{t}=\hat{h}_{t} / \mathcal{L}$

$A=\hat{A} / R^{2}$

$A_{a}=\hat{A}_{a} / R^{2}$

$A_{d}=\hat{A}_{d} / R^{2}$

$\mathcal{E}$

$\mathcal{P}$

$\Phi$

TABLE 2. List of the dimensionless parameters and variables of the problem.

and

$$
A r=\frac{\rho_{d} \Delta \rho g R^{3}}{\mu_{d}^{2}}, \quad \mathcal{R}=\frac{\rho_{a}}{\rho_{d}}, \quad M=\frac{\mu_{d}}{\mu_{a}},
$$

are the Archimedes number, $A r$, the density ratio, $\mathcal{R}$, and the viscosity ratio, $M$, respectively. The Archimedes number represents the ratio between buoyancy and viscous forces. It is equivalent to the Reynolds number defined on our characteristic axial velocity scale $U$, since $A r=R e_{U}=\rho_{d} U R / \mu_{d}$. The dimensionless parameters and variables of the problem are listed in table 2 .

In our analysis, we assume an elongated Taylor drop, i.e. $\varepsilon \ll 1$, with a region of constant drop thickness that extends over most of the length $\mathcal{L}$. In this limit, the axial derivative of $u_{i}$ and the terms including the radial component of the velocity $v_{i}$ can be neglected from (2.6) and (2.7), provided that $M \gg \varepsilon$. Therefore, (2.6) and (2.7) reduce to a one-dimensional set of equations

$$
\begin{gathered}
\frac{1}{r} \frac{\mathrm{d}}{\mathrm{d} r}\left(r \frac{\mathrm{d} u_{d}}{\mathrm{~d} r}\right)=\frac{\mathrm{d} p}{\mathrm{~d} z}+\frac{1}{1-\mathcal{R}}, \quad r \in[\delta, 1], \\
\frac{1}{M} \frac{1}{r} \frac{\mathrm{d}}{\mathrm{d} r}\left(r \frac{\mathrm{d} u_{a}}{\mathrm{~d} r}\right)=\frac{\mathrm{d} p}{\mathrm{~d} z}+\frac{\mathcal{R}}{1-\mathcal{R}}, \quad r \in[0, \delta],
\end{gathered}
$$

where $\partial p_{i} / \partial r=0$. Both fluids share the same pressure gradient, $\mathrm{d} p_{d} / \mathrm{d} z=\mathrm{d} p_{a} / \mathrm{d} z=$ $\mathrm{d} p / \mathrm{d} z$. Since the normal stress at the fluid-fluid interface is continuous, we find that the pressure difference between the fluids is controlled by the Bond number, $\mathrm{Bo}$, as follows:

$$
p_{d}-p_{a}=\frac{1}{\delta} \frac{\varepsilon}{B o}, \quad \text { with } B o=\frac{\Delta \rho g R^{2}}{\sigma}
$$

where $\sigma$ is the surface tension and $\delta=\hat{\delta} / R$ is the dimensionless drop thickness.

For elongated drops, the lubrication model (2.10) holds because the nonlinear terms on the left-hand side of (2.6) and (2.7) vanish. The model applies even at finite $A r$ if the viscous scaling chosen for defining the velocity scale $U$ remains representative of the physics of the problem. We only consider cases where the descending fluid is 


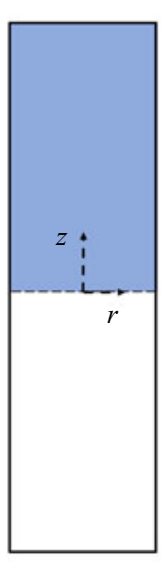

$t=0$

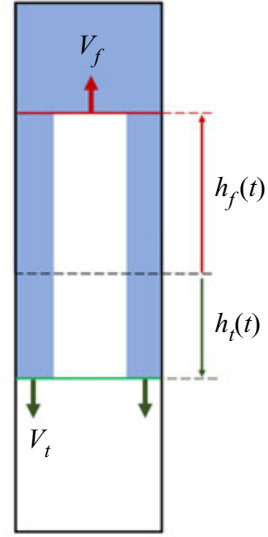

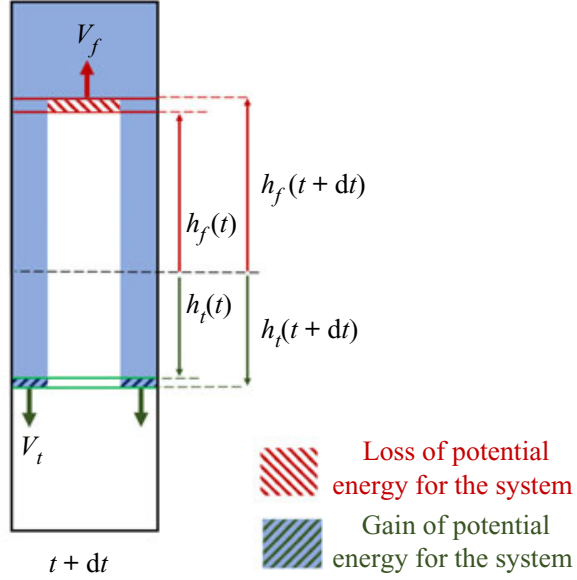

FIGURE 2. View of the axial cross-section of the lock-exchange problem in a vertical pipe $(z-r$ plane) in dimensionless coordinates. The ascending front (red); the descending tail (green); the gain of potential energy area (green diagonal stripes); and the loss of potential energy (red diagonal stripes).

heavier compared with the ascending fluid, $\rho_{d}>\rho_{a}$, and, therefore, $\mathcal{R}<1$. This choice is motivated by the problem of buoyancy-driven conduit flow in persistently degassing volcanoes and slug flow in vertical conduits more generally.

\subsection{Velocity profiles and front speed}

In this section, we derive analytical expressions for the velocity profiles, as well as for the ascent and descent speeds of the Taylor drop (illustrated in figure 2). After the release of the lock, the fluids start moving due to buoyancy: the lighter fluid penetrates into the heavier fluid at speed $V_{f}$ while the heavier fluid displaces the lighter fluid at speed $V_{t}$. Using a lubrication approximation, we conceptualize the Taylor drop as a region of core-annular flow with uniform thickness $\delta$ and length $\left|h_{f}\right|+\left|h_{t}\right|$, as sketched in figure 2 .

We integrate $(2.10 a)$ and $(2.10 b)$ subject to the following conditions.

(i) The pipe wall is no-slip and the radial derivative of velocity is zero at the pipe centre,

$$
u_{d}(1)=0, \quad \frac{\mathrm{d}}{\mathrm{d} r} u_{a}(0)=0 .
$$

(ii) Velocity and shear stress are continuous at the fluid-fluid interface,

$$
u_{d}(\delta)=u_{a}(\delta), \quad \frac{\mathrm{d}}{\mathrm{d} r} u_{d}(\delta)=\frac{1}{M} \frac{\mathrm{d}}{\mathrm{d} r} u_{a}(\delta) .
$$

We obtain the velocity profiles

$$
\begin{gathered}
u_{d}(r)=\frac{P}{4}\left(r^{2}-1\right)-\frac{\delta^{2}}{2} \log r, \quad r \in[\delta, 1], \\
u_{a}(r)=M \frac{P-1}{4}\left(r^{2}-\delta^{2}\right)+\frac{P}{4}\left(\delta^{2}-1\right)-\frac{\delta^{2}}{2} \log \delta, \quad r \in[0, \delta],
\end{gathered}
$$



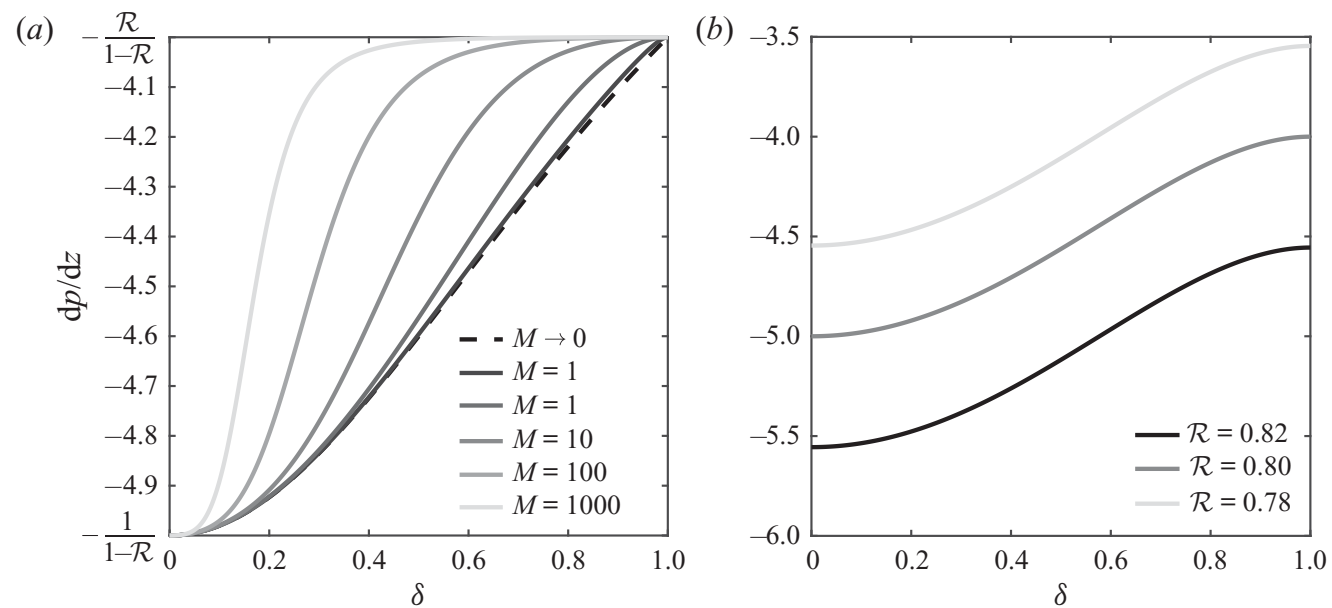

FIGURE 3. (a) Dimensionless pressure gradient $\mathrm{d} p / \mathrm{d} z$ as a function of the drop thickness $\delta$ and the viscosity ratio $M$ for $\mathcal{R}=0.8$. (b) Dimensionless pressure gradient as a function of the drop thickness $\delta$ and the density ratio $\mathcal{R}$ for $M=1$.

where $P=\mathrm{d} p / \mathrm{d} z+1 /(1-\mathcal{R})$ is the dimensionless driving force of the ascending fluid. We can eliminate the pressure gradient from $(2.14 a)$ and (2.14b) using the exchange-flow condition (2.2),

$$
\int_{\delta}^{1} 2 \pi r u_{d} \mathrm{~d} r+\int_{0}^{\delta} 2 \pi r u_{a} \mathrm{~d} r=0,
$$

and find that $P$ is a function of the drop thickness and the viscosity ratio

$$
P=\delta^{2} \frac{2\left(\delta^{2}-1\right)-M \delta^{2}}{\delta^{4}-1-M \delta^{4}} .
$$

This relation clarifies how the vertical pressure gradient $\mathrm{d} p / \mathrm{d} z$ changes with $\delta, M$ and $\mathcal{R}$ under exchange-flow condition, see figure 3 . As expected, the magnitude of the driving force increases with the density difference between the fluids, see figure 3(b).

When the drop thickness vanishes for $\delta \rightarrow 0$, the pressure gradient approaches the single-phase limit balancing the gravitational force of the descending fluid, $\mathrm{d} p / \mathrm{d} z \rightarrow$ $-1 /(1-\mathcal{R})$. In the opposing limit of $\delta \rightarrow 1, \mathrm{~d} p / \mathrm{d} z$ balances the gravitational force of the ascending fluid.

We obtain the ascent and descent speeds by integrating the velocity profiles

$$
V_{f}=\frac{1}{\pi \delta^{2}} \int_{0}^{\delta} 2 \pi r u_{a} \mathrm{~d} r \quad \text { and } \quad V_{t}=\frac{1}{\pi\left(1-\delta^{2}\right)} \int_{\delta}^{1} 2 \pi r u_{d} \mathrm{~d} r,
$$

yielding

$$
\begin{aligned}
& V_{f}=-\frac{\delta^{2}}{8} \frac{4\left[1+(M-1) \delta^{4}\right] \log \delta+(4-3 M) \delta^{4}+4(M-2) \delta^{2}-M+4}{1+(M-1) \delta^{4}}, \\
& V_{t}=-\frac{\delta^{4}}{8} \frac{4\left[1+(M-1) \delta^{4}\right] \log \delta+(4-3 M) \delta^{4}+4(M-2) \delta^{2}-M+4}{\left(M \delta^{4}-\delta^{4}+1\right)\left(\delta^{2}-1\right)} .
\end{aligned}
$$

The estimated speeds, $V_{f}$ and $V_{t}$, are functions of the drop thickness and the viscosity ratio only as plotted in figure 4 . The two curves quantify how much $V_{f}$ and $V_{t}$ change with $\delta$ 

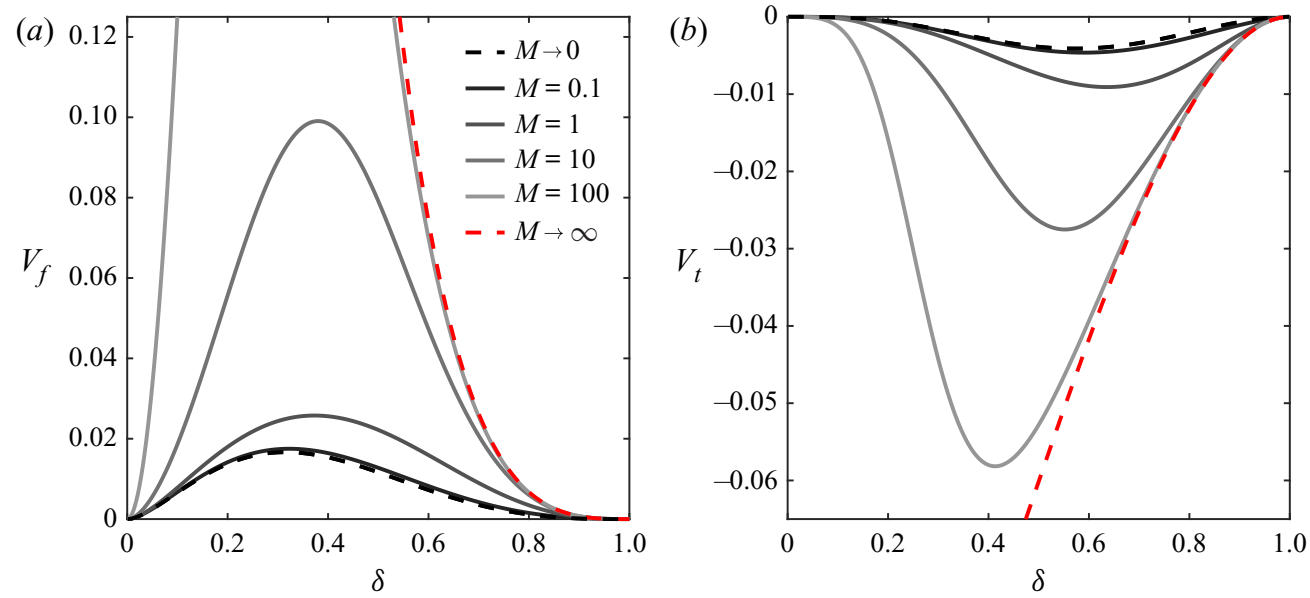

FIgURE 4. Ascent speed $V_{f}$, (2.18) and decent speed $V_{t}$ (2.19), as a function of the drop thickness $\delta$ and the viscosity ratio $M$.

while satisfying the exchange-flow condition. The two speeds are related through (2.15) as

$$
A_{a} V_{f}+A_{d} V_{t}=0
$$

where

$$
A_{a}=\pi \delta^{2}, \quad A_{d}=\pi\left(1-\delta^{2}\right),
$$

are the dimensionless cross-sectional area of the ascending and the descending fluids, respectively. Therefore, the two speeds and areas satisfy the following relation:

$$
\frac{V_{t}}{V_{f}}=-\frac{A_{a}}{A_{d}}=-\frac{\delta^{2}}{1-\delta^{2}} .
$$

The ascent speed is maximal for $M \rightarrow \infty$. In this limit, the viscosity of the descending fluid greatly exceeds that of the ascending fluid. As a consequence, the fluid-fluid interface resembles a free surface as evident when applying the limit $M \rightarrow \infty$ to the interface condition $(2.13 a, b) \mathrm{d} u_{d} /\left.\mathrm{d} r\right|_{\delta} \approx 0$. We refer to this case as the free-surface limit, where the asymptotic speeds are

$$
\begin{gathered}
V_{f}(\delta, M \rightarrow \infty)=\frac{\delta^{4}(1-\log \delta)+1-4 \delta^{2}}{8 \delta^{2}}, \\
V_{t}(\delta, M \rightarrow \infty)=\frac{\delta^{4}(3-4 \log \delta)+1-4 \delta^{2}}{8\left(\delta^{2}-1\right)} .
\end{gathered}
$$

The other asymptotic limit is $M \rightarrow 0$. In this case, the drop is so viscous that it can be approximated as a rigid body. We refer to this limit as the rigid-core limit, which is characterized by

$$
\begin{gathered}
V_{f}(\delta, M \rightarrow 0)=-\frac{\delta^{2}\left(1-\delta^{2}+\log \delta+\delta^{2} \log \delta\right)}{2\left(\delta^{2}+1\right)}, \\
V_{t}(\delta, M \rightarrow 0)=\frac{\delta^{4}\left(1+\log \delta-\delta^{2}-\delta^{2} \log \delta\right)}{2\left(\delta^{4}-1\right)} .
\end{gathered}
$$




\section{Energy analysis}

\subsection{Energy balance}

Here, we formulate the energy balance for the Taylor drop formed from a lock-exchange configuration as conceptualized in figure 2. Our analysis applies once the Taylor drop has formed and elongated sufficiently such that the lubrication approximation derived in $\S 2$ holds. We do not consider transient behaviour immediately after the release of the lock.

We start from the energy balance formulated by Dussan (1975) for a closed system filled with two immiscible fluids that occupy the volume $\hat{\mathcal{V}}=\hat{\mathcal{V}}_{a} \cup \hat{\mathcal{V}}_{d}$ and are separated by the fluid-fluid interface $\hat{\Sigma}$ given by

$$
\frac{\mathrm{d}}{\mathrm{d} \hat{t}}\left[\hat{\mathcal{E}}+\hat{\mathcal{P}}+\oint_{\hat{\Sigma}} \sigma \mathrm{d} \hat{S}\right]=\oint_{\partial \hat{\Sigma}} \sigma \boldsymbol{t} \cdot \hat{\boldsymbol{U}} \mathrm{d} \hat{\ell}+\int_{\partial \hat{\mathcal{V}}} \hat{\boldsymbol{u}} \cdot(\hat{\boldsymbol{\tau}} \cdot \boldsymbol{n}) \mathrm{d} \hat{S}-\hat{\Phi},
$$

where $\hat{\mathcal{E}}, \hat{\mathcal{P}}, \oint_{\hat{\Sigma}} \sigma \mathrm{d} \hat{S}$ and $\hat{\Phi}$ are the kinetic, the potential, the surface energy and the total viscous dissipation of the system, respectively, while $\hat{U}, \hat{\tau}$ and $t$ are the velocity of the contact line, the shear stress tensor and the unit vector normal to $\partial \hat{\Sigma}$ on $\hat{\Sigma}$. The first integral on the right-hand side of (3.1) accounts for the work done by the contact line $\partial \hat{\Sigma}$. The second integral is the work of the traction on the pipe wall $\partial \hat{\mathcal{V}}$, which is zero in our case since the motion is not forced from the boundary (Joseph, Nguyen \& Beavers 1984). The last term is the viscous dissipation produced in the whole fluid domain.

If we non-dimensionalize (3.1) based on the characteristic scales defined in $(2.5 a-f)$, we obtain

$$
\frac{\mathrm{d}}{\mathrm{d} t}\left[\frac{1}{R i} \mathcal{E}+\mathcal{P}+(1-\mathcal{R}) \frac{\varepsilon}{B o} \oint_{\Sigma} \mathrm{d} S\right]=(1-\mathcal{R}) \frac{\varepsilon}{B o} \oint_{\partial \Sigma} t \cdot U \mathrm{~d} \ell-(1-\mathcal{R}) \Phi,
$$

where $R i$ is the Richardson number of the Taylor drop defined as

$$
R i=\frac{g \mathcal{L}}{U^{2}} .
$$

The Richardson number represents the ratio between potential energy, $\rho_{a} g \mathcal{L}$, and kinetic energy, $\rho_{a} U^{2}$. As described in $\S 2.2$, we are interested in elongated drops, $\varepsilon \ll 1$, and, therefore, we can eliminate the surface energy and the work of the contact line terms from (3.2). With this, we obtain a simplified energy balance

$$
\frac{\mathrm{d}}{\mathrm{d} t}\left[\frac{1}{R i} \mathcal{E}+\mathcal{P}\right]=-(1-\mathcal{R}) \Phi
$$

where energy of the system is approximated by the sum of the kinetic and potential energy and is dissipated only through viscous dissipation. This simplification holds in systems with negligible surface tension, $B o \gg \varepsilon$.

Based on the scaling chosen in this paper, see $(2.5 a-f)$, we can rewrite $R i$ as

$$
R i=\frac{1}{(1-\mathcal{R}) \varepsilon A r},
$$

and further simplify the energy balance in the limit of $R i \rightarrow \infty$ as

$$
\frac{\mathrm{d}}{\mathrm{d} t} \mathcal{P}=-(1-\mathcal{R}) \Phi
$$


In this case, the dynamics of the system is controlled by the interplay between the time rate of potential energy and viscous dissipation.

\subsubsection{Kinetic energy}

The kinetic energy of the system is defined as

$$
\hat{\mathcal{E}}=\int_{\hat{\mathcal{V}}_{d}} \frac{1}{2} \rho_{d} \hat{u}_{d}^{2} \mathrm{~d} \hat{V}+\int_{\hat{\mathcal{V}}_{a}} \frac{1}{2} \rho_{a} \hat{u}_{a}^{2} \mathrm{~d} \hat{V}
$$

We only consider the kinetic energy in the drop region $\mathcal{L}$ where the analytical velocity profiles (2.14) apply. We neglect the kinetic energy in the region above and below the Taylor drop because the aspect ratio of the pipe is very small, $R / L \ll 1$. This simplification is equivalent to assuming that the regions of pure fluids above and below the drop act as infinite reservoirs. Using (2.14) in (3.7) and integrating over the region $\mathcal{L}$ of constant thickness $\delta$, we obtain the dimensionless kinetic energy

$$
\mathcal{E}=\pi\left(h_{f}-h_{t}\right)\left[\frac{1}{1-\mathcal{R}} \int_{\delta}^{1} r u_{d}^{2} \mathrm{~d} r+\frac{\mathcal{R}}{1-\mathcal{R}} \int_{0}^{\delta} r u_{a}^{2} \mathrm{~d} r\right],
$$

where we express the height of the ascending front and the descending tail as

$$
h_{f}=V_{f} t \quad \text { and } \quad h_{t}=V_{t} t
$$

Finally, we obtain

$$
\begin{aligned}
\mathcal{E}= & \pi \frac{\left(V_{f}-V_{t}\right) t}{96}\left[\left(\frac{1}{\mathcal{R}-1}-\delta^{6}-3 \delta^{4}-3 \delta^{2}\right) P^{2}+12(\log \delta)^{2}\left(\delta^{6}+\frac{\delta^{4}}{\mathcal{R}-1}\right)\right. \\
& \left.-6 \delta^{2} P \log \delta\left(\delta^{4}-2 \delta^{2}-\frac{1}{\mathcal{R}-1}\right)\right] .
\end{aligned}
$$

\subsubsection{Potential energy}

The potential energy of the system at the time $\hat{t}$ is defined as

$$
\hat{\mathcal{P}}=\int_{\hat{\mathcal{V}}_{d}} \rho_{d} g \hat{z}_{d} \mathrm{~d} \hat{V}+\int_{\hat{\mathcal{V}}_{a}} \rho_{a} g \hat{z}_{a} \mathrm{~d} \hat{V}
$$

Since we assume constant thickness $\hat{\delta}$, (3.11) becomes

$$
\hat{\mathcal{P}}=\rho_{d} g\left[\hat{A}_{d} \int_{\hat{h}_{t}}^{\hat{h}_{f}} \hat{z}_{d} \mathrm{~d} \hat{z}+\hat{A} \int_{\hat{h}_{f}}^{L} \hat{z}_{d} \mathrm{~d} \hat{z}\right]+\rho_{a} g\left[\hat{A}_{a} \int_{\hat{h}_{t}}^{\hat{h}_{f}} \hat{z}_{a} \mathrm{~d} \hat{z}+\hat{A} \int_{-L}^{\hat{h}_{t}} \hat{z}_{d} \mathrm{~d} \hat{z}\right],
$$

and we obtain

$$
\hat{\mathcal{P}}=\frac{1}{2} g \hat{A}_{d} \hat{h}_{t}^{2}\left(\rho_{d}-\rho_{a}\right)-\frac{1}{2} g \hat{A}_{a} \hat{h}_{f}^{2}\left(\rho_{d}-\rho_{a}\right)+\frac{1}{2}\left(\rho_{d}-\rho_{a}\right) g \hat{A} L^{2},
$$

where $\hat{A}=\pi R^{2}, \hat{A}_{a}=\pi \hat{\delta}^{2}$ and $\hat{A}_{d}=\pi\left(R^{2}-\hat{\delta}^{2}\right)$ are the pipe cross-section and the cross-sectional areas occupied by the ascending and the descending fluids, respectively. 
Using $(3.9 a, b)$ and recasting the equation in terms of dimensionless variables, yields

$$
\mathcal{P}=\frac{1}{2}\left(A_{d} V_{t}^{2}-A_{a} V_{f}^{2}\right) t^{2}+\frac{\pi}{2} L_{*}^{2},
$$

where the dimensionless areas $A_{a}$ and $A_{d}$ are defined in $(2.21 a, b)$ and $L_{*}=L / \Delta \rho g U^{2} R^{2}$ is a constant.

Before the removal of the lock at time $t=0$, the velocity field is zero everywhere and the potential energy is maximal, see figure 2. Between the time instants $t$ and $t+\mathrm{d} t$, the light fluid invades a region $\mathrm{d} \mathcal{V}_{f}$ that was previously occupied by the heavy fluid. Although the potential energy of the ascending fluid increases due to the increased height of the column, the replacement of the heavy fluid by the light fluid results in a loss of $\mathcal{P}$. At the descending tail, instead, the heavy fluid occupies a region $\mathrm{d} \mathcal{V}_{t}$ that was previously occupied by the lighter fluid, resulting in a gain of $\mathcal{P}$. These two competing mechanisms are evident when examining the time derivative of the potential energy (keeping $\delta$ fixed)

$$
\left(\frac{\partial \mathcal{P}}{\partial t}\right)_{\delta}=(\underbrace{A_{d} V_{t}^{2}}_{\text {gain }} \underbrace{-A_{a} V_{f}^{2}}_{\text {loss }}) t .
$$

The rate at which $\mathcal{P}$ increases scales with $A_{d} V_{t}^{2}$ while the rate of energy loss scales with $A_{a} V_{f}^{2}$.

\subsubsection{Viscous dissipation}

We only consider the viscous dissipation in the region $\mathcal{L}$. Viscous dissipation is defined as

$$
\hat{\Phi}=\int_{\hat{\mathcal{V}}_{d}} \mu_{d}\left(\frac{\partial \hat{u}_{d}}{\partial \hat{r}}\right)^{2} \mathrm{~d} \hat{V}+\int_{\hat{\mathcal{V}}_{a}} \mu_{a}\left(\frac{\partial \hat{u}_{a}}{\partial \hat{r}}\right)^{2} \mathrm{~d} \hat{V}
$$

or, in term of dimensionless variables,

$$
\Phi=\left(h_{f}-h_{t}\right)\left[\int_{\delta}^{1} 2 \pi r\left(\frac{\mathrm{d} u_{d}}{\mathrm{~d} r}\right)^{2} \mathrm{~d} r+\frac{1}{M} \int_{0}^{\delta} 2 \pi r\left(\frac{\mathrm{d} u_{a}}{\mathrm{~d} r}\right)^{2} \mathrm{~d} r\right]
$$

After using the velocity profiles from (2.14) and our estimates for front and tail heights $(3.9 a, b)$, we obtain

$$
\Phi=2 \pi\left(V_{f}-V_{t}\right)\left[\frac{P}{16}\left(\delta^{2}-1\right)^{2}+\frac{\delta^{2}}{8}\left(\delta^{2}-1-2 \delta^{2} \log \delta\right)\right] t .
$$

We can further simplify (3.18) using (2.18), (2.19) and (2.22) yielding

$$
\Phi=A_{a} V_{f}\left(V_{f}-V_{t}\right) t
$$

\subsection{Time evolution of the drop thickness}

We now use the energy balance (3.4) to track the evolution of the drop thickness over time $\delta(t)$. We start by observing that the kinetic and potential energy, (3.10) and (3.14), and the viscous dissipation (3.18), depend only on the drop thickness $\delta$, the time $t$ and 
a finite number of material parameters including the viscosity and density ratios and the Richardson number. Therefore, we can recast the energy balance as follows:

$$
\left[\frac{1}{R i} \frac{\partial \mathcal{E}}{\partial \delta}+\frac{\partial \mathcal{P}}{\partial \delta}\right] \frac{\mathrm{d} \delta}{\mathrm{d} t}+\frac{1}{R i} \frac{\partial \mathcal{E}}{\partial t}+\frac{\partial \mathcal{P}}{\partial t}=-(1-\mathcal{R}) \Phi .
$$

Since all the derivatives of the kinetic and potential energy $(\partial / \partial \delta$ and $\partial / \partial t)$ can be computed analytically, (3.20) is an ordinary differential equation for $\delta(t)$ in the form

$$
f(\delta, t) \frac{\mathrm{d} \delta}{\mathrm{d} t}+g(\delta, t)=-(1-\mathcal{R}) \Phi(\delta, t),
$$

where

$$
\begin{aligned}
& f(\delta, t)=\frac{1}{R i} \frac{\partial \mathcal{E}}{\partial \delta}+\frac{\partial \mathcal{P}}{\partial \delta}, \\
& g(\delta, t)=\frac{1}{R i} \frac{\partial \mathcal{E}}{\partial t}+\frac{\partial \mathcal{P}}{\partial t} .
\end{aligned}
$$

The analytical expression for $g(\delta, t)$ and the computational procedure for $f(\delta, t)$ are detailed in appendix A. Equation (3.21) can then be solved for any initial condition in the domain $\delta \in[0,1]$ for $t \in\left[t_{0}, \infty\right]$.

When $R i \rightarrow \infty$ we can simplify (3.21) to

$$
\frac{\partial \mathcal{P}}{\partial \delta} \frac{\mathrm{d} \delta}{\mathrm{d} t}+\frac{\partial \mathcal{P}}{\partial t}=-(1-\mathcal{R}) \Phi
$$

The full analytical expressions are reported in appendix B. For sake of physical interpretation, we focus on three end-member cases when solving (3.24) while distinguishing between the non-dissipative $(\Phi=0)$ and the dissipative regimes $(\Phi \neq 0)$ :

(i) two isoviscous fluids, $M=1$,

$$
\frac{\mathrm{d} \delta}{\mathrm{d} t}=-\frac{\delta\left(\delta^{2}-1\right)\left(2 \delta^{2}-\psi\right)\left(\delta^{4}-4 \delta^{4}+4 \log \delta+3\right)}{t\left[\left(24 \delta^{4}-40 \delta^{2}+12\right) \log \delta 14 \delta^{8}-62 \delta^{6}+97 \delta^{4}-62 \delta^{2}+13\right]} ;
$$

(ii) the rigid-core limit, $M \rightarrow 0$,

$$
\frac{\mathrm{d} \delta}{\mathrm{d} t}=-\frac{\delta\left(\delta^{4}-1\right)\left(2 \delta^{2}-\psi\right)\left[\left(\delta^{2}+1\right) \log \delta-\delta^{2}+1\right]}{t\left[\left(\delta^{2}+1\right)^{2}\left(6 \delta^{4}-10 \delta^{2}+3\right) \log \delta-4 \delta^{8}+3 \delta^{6}+12 \delta^{4}-15 \delta^{2}+4\right]} ;
$$

(iii) the free-surface limit, $M \rightarrow \infty$,

$$
\frac{\mathrm{d} \delta}{\mathrm{d} t}=-\frac{\delta\left(\delta^{2}-1\right)\left(2 \delta^{2}-\psi\right)\left(4 \delta^{4} \log \delta-3 \delta^{4}+4 \delta^{2}-1\right)}{t\left[\left(24 \delta^{8}-40 \delta^{6}+12 \delta^{4}\right) \log \delta-10 \delta^{8}+26 \delta^{6}-19 \delta^{4}+2 \delta^{2}+1\right]},
$$

where

$$
\begin{gathered}
\psi=1 \quad \text { when } \Phi=0, \\
\psi=\mathcal{R} \quad \text { when } \Phi \neq 0 .
\end{gathered}
$$

Note that (3.25), (3.26) and (3.27) have been obtained taking the asymptotic limits of (B 2) for $M \rightarrow 1, M \rightarrow 0$ and $M \rightarrow \infty$, respectively. In the absence of dissipation, we can 
compute the steady-state solutions of (3.25), (3.26) and (3.27) in the range $\delta \in[0,1]$ by computing the roots of $\mathrm{d} \delta / \mathrm{d} t=0$. We obtain three steady-state solutions

$$
\delta_{\infty}=\frac{\sqrt{2}}{2}, \quad \delta_{\infty}=0 \quad \text { and } \quad \delta_{\infty}=1 .
$$

Equivalently, in the dissipative regime, the three steady-state solutions are

$$
\delta_{\infty}=\frac{\sqrt{2 \mathcal{R}}}{2}, \quad \delta_{\infty}=0 \quad \text { or } \quad \delta_{\infty}=1 .
$$

\section{Discussion}

\subsection{Transient evolution and ascent speed of the Taylor drop}

We obtain the transient evolution of the Taylor drop by solving the ordinary differential equation (3.21) in time for an arbitrary initial condition. We initialize the problem with a drop of thickness $\delta \in[0,1]$ and compute the time evolution in the interval $\left[t_{0}, \infty\right]$. The computations are performed using the stiff solver ode15s of Matlab. The ordinary differential equation (3.21) is singular at $t=0$, because the model only applies once a Taylor drop has formed. We hence start our computation at $t_{0}=10$ to guarantee the uniqueness of the solution for arbitrary initial drop thickness, viscosity ratio and Richardson number. We focus on Richardson numbers in the range $R i \in[1, \infty]$ and present the time evolution of $\delta(t)$ for (i) the rigid-core limit, (ii) isoviscous fluids and (iii) the free-surface limit.

In the non-dissipative regime, the drop thickness converges to the stable solution $\delta_{\infty}=$ $\sqrt{2} / 2$ for an intermediate range of initial conditions, highlighted in yellow in figure 5 . Otherwise, the drop collapses to one of the two single-phase limits, $\delta \rightarrow 0$ and $\delta \rightarrow 1$, leading to a flow configuration that does not entail a Taylor drop and is hence beyond the scope of this study.

The transient dynamics is controlled by $M$ and $R i$. When $R i \rightarrow \infty$, a stable Taylor drop forms for initial thicknesses between $0.4<\delta<0.76$. The width of the stable region increases for $M \leq 1$ and widens up to $0<\delta<0.76$ in the free-surface limit. The free-surface limit is hence the most robust configuration and strongly favours the formation of a stable Taylor drop.

At steady state, both the ascending and the descending fluids occupy half of the cross-sectional area, $A_{a} / A_{d}=1$, and the energy balance (3.24) reduces to

$$
\frac{\partial \mathcal{P}}{\partial t}\left(\delta_{\infty}\right)=0, \quad \text { for } t \rightarrow \infty,
$$

revealing that the potential energy is constant with time. If we manipulate (4.1) using (2.22) and (3.15) we obtain

$$
-A_{a} V_{f}\left(V_{f}+V_{t}\right)=0, \quad \text { for } t \rightarrow \infty,
$$

and see that the ascent speed is equal to the descent speed, $V_{f}=-V_{t}$. This means that the loss in potential energy at the ascending front is balanced by the gain in potential energy at the descending tail in the non-dissipative regime, see (3.15) with $A_{a}=A_{d}$ and $V_{f}=-V_{t}$. The speeds $V_{f}$ and $V_{t}$ depend on the viscosity ratio and can be computed by evaluating 

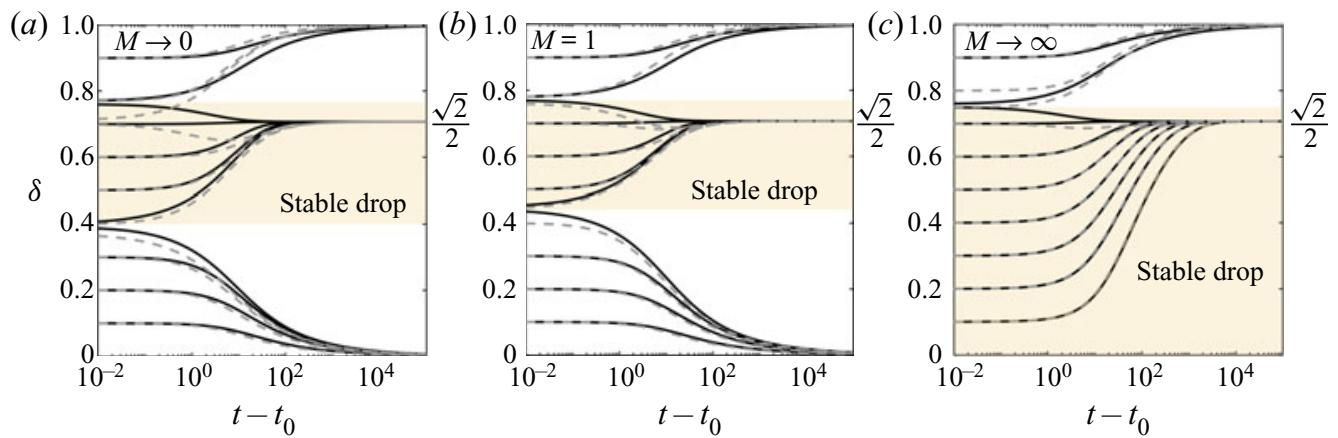

FIGURE 5. Transient evolution of the Taylor drop thickness as a function of time computed by solving the energy balance in the non-dissipative regime in the interval $t=\left[t_{0}, 10^{5}\right]$ with $t_{0}=10$. The plot shows the viscous limit $R i \rightarrow \infty$ (solid line) and the cases with $R i=1$ and $\mathcal{R}=0.8$ (dashed lines).

(2.18) and (2.19) at $\delta_{\infty}=\sqrt{2} / 2$ as

$$
\begin{gathered}
V_{f}=\frac{(6+2 M) \log 2-M-4}{48+16 M}, \\
V_{t}=-\frac{(6+2 M) \log 2-M-4}{48+16 M} .
\end{gathered}
$$

In the dissipative regime, the transient behaviour is qualitatively similar (see figure 6), but the steady-state solution is determined by the interplay of three mechanisms: the loss in potential energy at the ascending front; the gain in potential energy at the descending tail; and viscous dissipation. The energy balance (3.24) is

$$
\frac{\partial \mathcal{P}}{\partial t}\left(\delta_{\infty}\right)=-(1-\mathcal{R}) \Phi\left(\delta_{\infty}\right) \quad \text { for } t \rightarrow \infty,
$$

indicating that the potential energy decreases with time due to dissipation. Substituting (3.15) and (3.19) into (4.5) and using (2.22), we find that the steady-state solution is $\delta_{\infty}=$ $\sqrt{2 \mathcal{R}} / 2$. Since $\mathcal{R}<1$, the viscous dissipation causes the drop to shrink and flow faster than in the non-dissipative regime, see figure $7(a)$. In fact, the ascending fluid occupies less than half of the cross-section, $A_{a}\left(\delta_{\infty}\right) / A=\mathcal{R} / 2$, and, therefore, $V_{f}>\left|V_{t}\right|$, as evident from (2.22)

$$
\frac{V_{t}}{V_{f}}=-\frac{A_{a}}{A_{d}}=-\frac{\mathcal{R}}{2-\mathcal{R}}, \quad \text { with } \delta_{\infty}=\frac{\sqrt{2 \mathcal{R}}}{2},
$$

where $V_{f}(M, \mathcal{R})$ and $V_{t}(M, \mathcal{R})$ are calculated from (2.18) and (2.19). Note that the ascent speed is approximately one order of magnitude higher in the free-surface limit than in the rigid-core limit for almost the entire range of density ratios as shown in figure $7(b)$.

Our findings extend the classical results of Benjamin (1968) for horizontal gravity currents to the vertical configuration. Benjamin (1968) showed that an inviscid bubble propagating in a horizontal tube has a dimensionless thickness of $1 / 2$. The thickness reduces when dissipation is accounted for, as demonstrated by Shin, Dalziel \& Linden (2004). In this work, we observe the same qualitative trend for a liquid elongated drop formed in vertical pipe. 

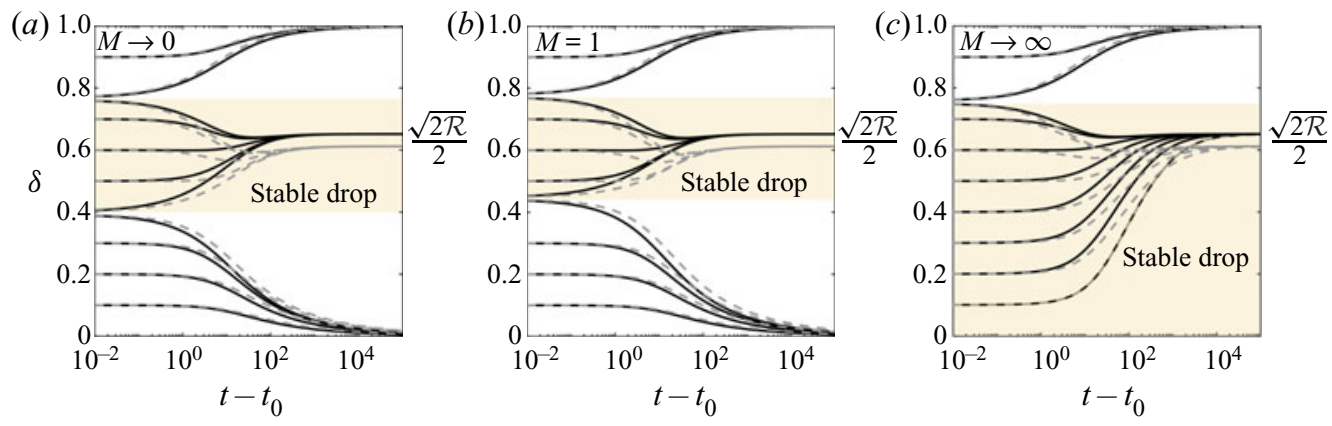

FIGURE 6. Transient evolution of the drop thickness as a function of time computed by solving the energy balance with viscous dissipation in the interval $t=\left[t_{0}, 10^{5}\right]$ with $t_{0}=10$. The plot shows the viscous limit $R i \rightarrow \infty$ and the effect of the density ratio $\mathcal{R}$. Solid lines have $\mathcal{R}=0.85$ and dashed lines have $\mathcal{R}=0.75$.
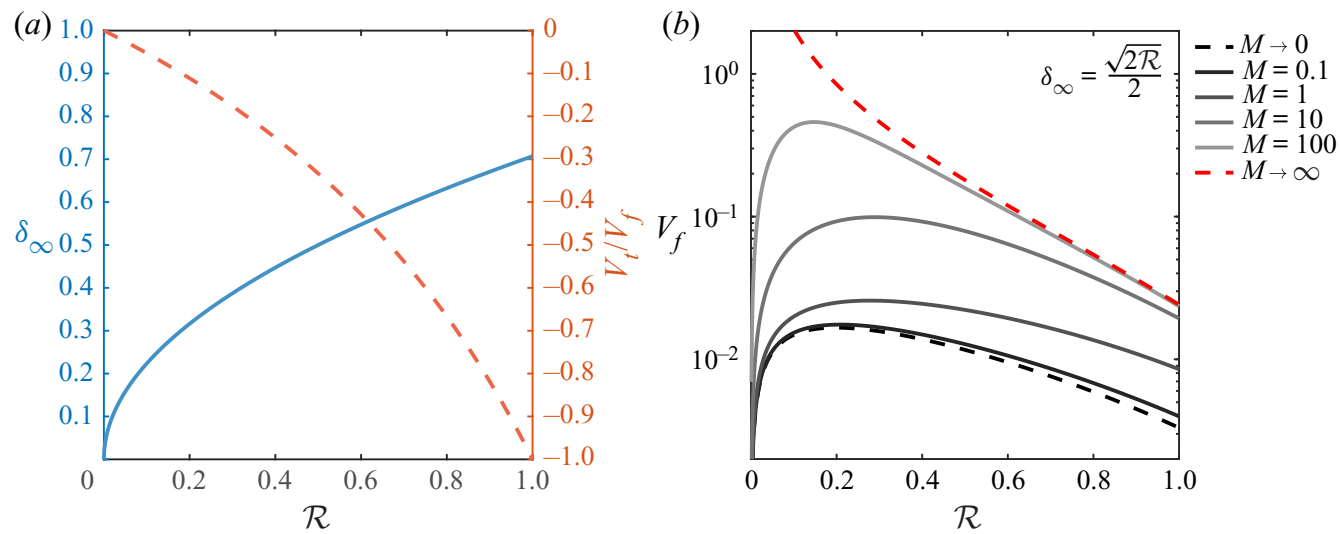

FIGURE 7. (a) Drop thickness and ratio of descent to ascent speed as a function of the density ratio for the dissipative regime. (b) Ascent speed $V_{f}$ as a function of the density and viscosity ratios for the dissipative regime.

\subsection{Validation against lock-exchange experiments}

We validate our model against the experiments by Stevenson \& Blake (1998) that measured the ascent speed of a Taylor drop formed from a lock-exchange configuration for different combinations of high-viscosity fluids. The experiments cover viscosity ratios of $M=30-O\left(10^{5}\right)$ at high Bond number, $B o \gg 1$. Here, we only consider experiments where a stable drop has formed to ensure that our model is applicable. For these experiments, the drops are elongated enough such that $\varepsilon \ll 1$ and the Archimedes number is sufficiently small, $A r=O\left(10^{-5}\right)-O\left(10^{-1}\right)$. We list all the details of the experiments used for the validation in table 3 .

Figure $8(a)$ shows that the measured ascent speed (solid circles) saturates around a constant value when the viscosity ratio is sufficiently large. Our model captures this trend well. When neglecting dissipation, our model slightly underestimates $V_{f}$, but it agrees well with the experiments in the dissipative regime. All the curves in figure 8(a) indicate that the drop's speed reaches a plateau when approaching the rigid-core and the 


$\begin{array}{lcccccc}\text { Reference } & \text { Type } & M & \mathcal{R} & A r & V_{f} & \delta_{\infty} \\ \text { Stevenson \& Blake (1998) } & \text { lock-exchange } & 29.36 & 0.88 & 2.91 \times 10^{-4} & 0.061 & 0.62 \\ & \text { lock-exchange } & 68.46 & 0.95 & 3.87 \times 10^{-5} & 0.065 & 0.61 \\ & \text { lock-exchange } & 1371.43 & 0.90 & 2.86 \times 10^{-5} & 0.067 & 0.61 \\ & \text { lock-exchange } & 1700.00 & 0.80 & 5.80 \times 10^{-2} & 0.061 & 0.62 \\ & \text { lock-exchange } & 1.85 \times 10^{4} & 0.80 & 2.07 \times 10^{-5} & 0.065 & 0.60 \\ & \text { lock-exchange } & 3.06 \times 10^{4} & 0.70 & 1.95 \times 10^{-3} & 0.062 & 0.62 \\ & \text { lock-exchange } & 3.46 \times 10^{4} & 0.70 & 1.52 \times 10^{-3} & 0.065 & 0.61 \\ \text { Stevenson \& Blake (1998) } & \text { long drop } & 1.46 \times 10^{5} & 0.70 & 8.66 \times 10^{-5} & 0.066 & 0.53 \\ & \text { long drop } & 4.12 \times 10^{4} & 0.70 & 1.08 \times 10^{-3} & 0.052 & 0.64 \\ & \text { long drop } & 44.95 & 0.88 & 3.91 \times 10^{-4} & 0.062 & 0.64 \\ \text { Goldsmith \& Mason (1962) } & \text { long drop } & 2.11 & 0.91 & 1.24 \times 10^{-1} & 0.030 & 0.60 \\ & \text { long drop } & 0.91 & 0.82 & 10.89 & 0.007 & 0.75 \\ & \text { long drop } & 0.12 & 0.80 & 13.35 & 0.003 & 0.74 \\ & \text { long drop } & 0.02 & 0.80 & 13.35 & 0.003 & 0.73\end{array}$

TABLE 3. Dimensionless numbers, ascent speed and drop thickness of the experiments used for validating the model. The data of Stevenson \& Blake (1998) are obtained with miscible fluids, $B o \gg 1$, while the data of Goldsmith \& Mason (1962) are for immiscible fluids with $B o \approx 1.3$.

free-surface limits. The transition between the two asymptotic behaviours occurs around $M=O(1)-O(10)$.

The model also approximately matches the measured drop thicknesses in the dissipative regime as demonstrated in figure $8(b)$. Stevenson \& Blake (1998) observed that the drops evolve to a uniform thickness around $\delta_{\infty} \approx 0.6$. Our energy analysis predicts $\delta_{\infty}$ within $\pm 15 \%$ of the experimental value in the range of $\mathcal{R} \approx 0.7-0.9$, see figure 9 . We consider this agreement satisfactory, particularly considering that the model is purely predictive and does not require any fitting parameters. For a more complete test of our model results, more experiments at intermediate $M$ would be valuable to better characterize the transition between drop behaviour in the rigid-core and the free-surface limits.

\subsection{Isolated Taylor drop}

We refer to a Taylor drop as isolated when the tail is a closed interface and does not touch the wall. In addition to lock-exchange experiments, Stevenson \& Blake (1998) injected a long drop at the bottom of the vertical pipe. They showed that the ascent speed of an isolated Taylor drop is indistinguishable from a drop formed from the lock-exchange configuration, see the solid circles in figure 8 . Motivated by these observations, we compare the predictions of the ascent speed $V_{f}$ for a lock-exchange configuration with experimental data of isolated Taylor drops. Among the experiments of isolated Taylor drops available in the literature we use data from Stevenson \& Blake (1998) and Goldsmith \& Mason (1962), see table 3. Although Kurimoto et al. (2013) and Hayashi et al. (2011) measured the ascent speed of Taylor drops covering a wide range of $A r=O(10)-O(5000)$, $M=O(0.1)-O(10), B o=O(1)-O(10)$ and $R \approx 0.8$, our model does not apply to their experiments, because the drops are not long enough to fulfil the requirement that $\varepsilon \ll 1$ and $\varepsilon A r \ll 1$. 

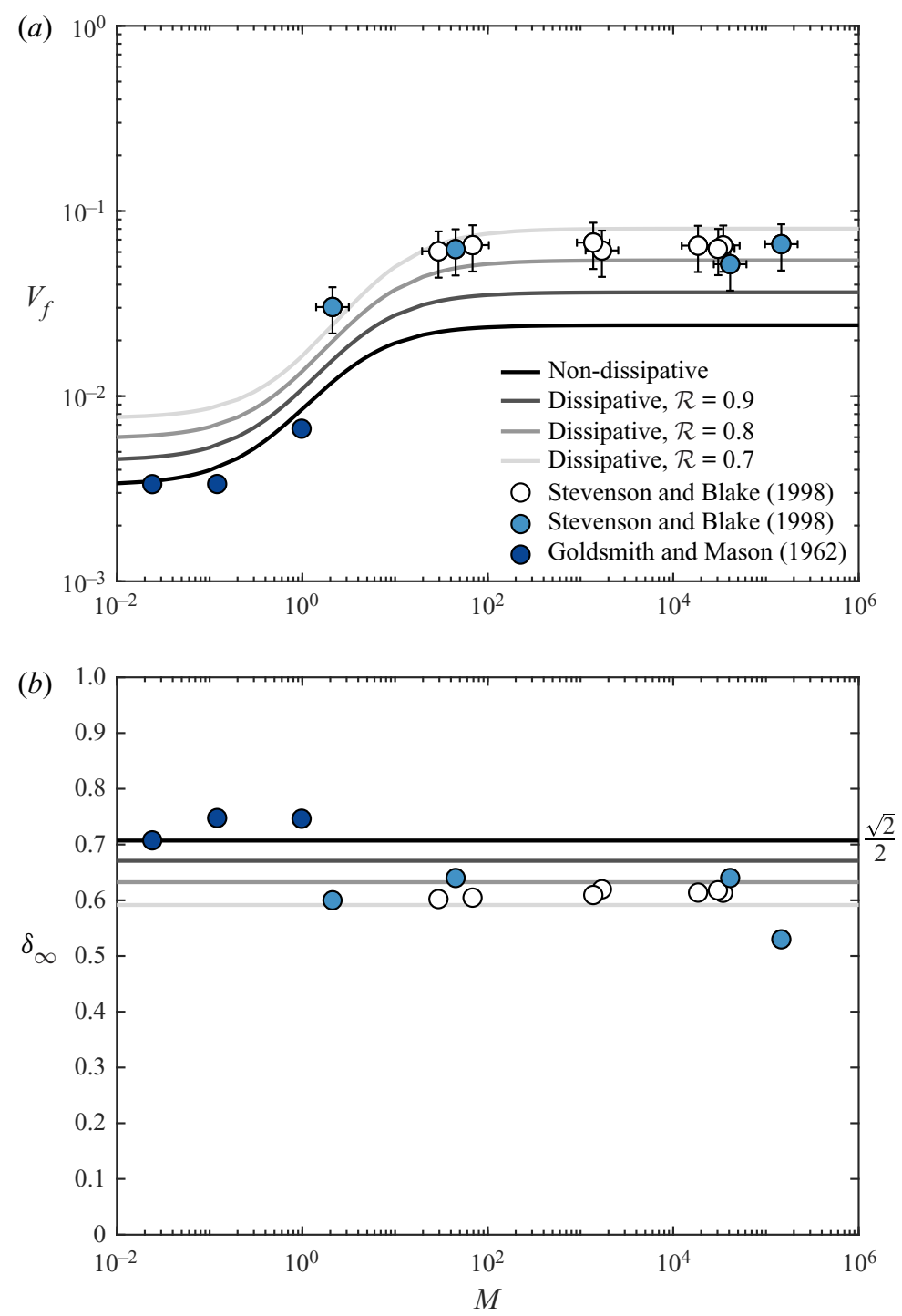

FIgURE 8. (a) Ascent speed $V_{f}$ as a function of the viscosity ratio $M$. (b) Taylor drop thickness at the steady-state $\delta_{\infty}$ as a function of the viscosity ratio $M$. The solid line is the energy balance (3.21). The experimental data from Stevenson \& Blake (1998) are lock-exchange experiments (the empty circles) and long drops (solid circles). The data from Goldsmith \& Mason (1962) are elongated drops.

Figure 9 compares theoretical and experimental drop thicknesses, demonstrating that our model agrees well with observations. The $\delta_{\infty}$ measured by Goldsmith \& Mason (1962) is very close to $\sqrt{2} / 2$ and it is within $\pm 15 \%$ error if we account for viscous dissipation. More interestingly, we can see in figure 8 that the isolated drop data confirms the existence of the two plateaus at $M \rightarrow 0$ and $M \rightarrow \infty$.

The insight that our model also describes isolated Taylor drops suggests that we can apply it to slug flow, defined as an intermittent flow pattern that consists of a train of isolated Taylor bubbles or drops. It is common practice in the literature (Wallis 1969; 


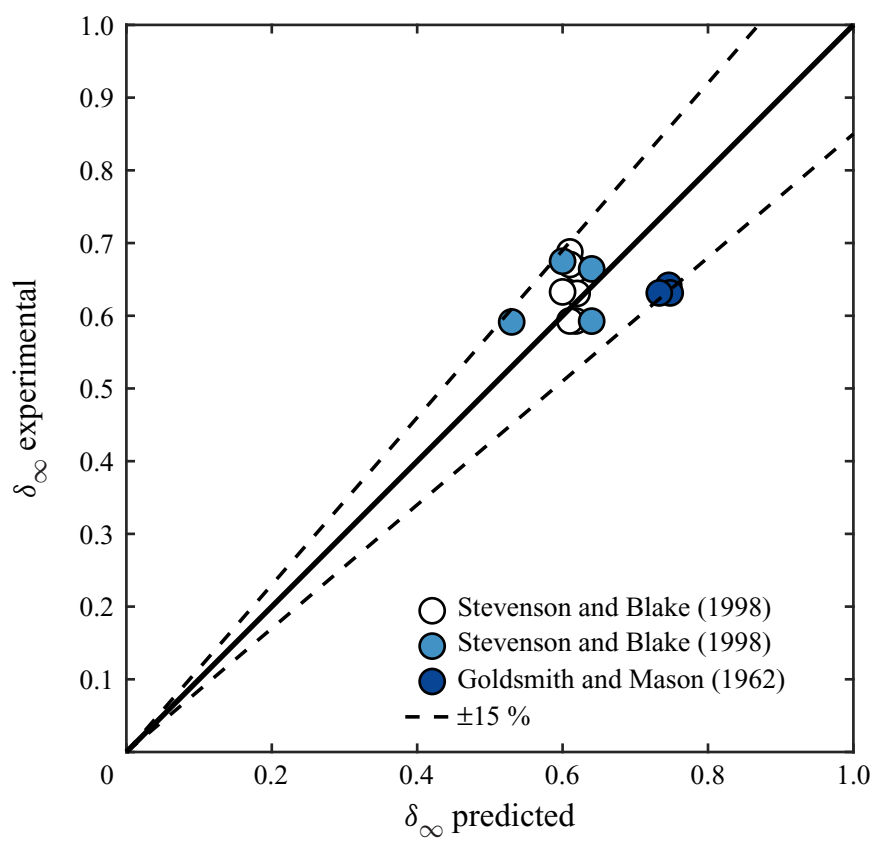

FIGURE 9. Comparison between the computed drop thickness at steady state $\delta_{\infty}$ and experimental measurements of drop thickness in the dissipative regime. The experimental data from Stevenson \& Blake (1998) are lock-exchange experiments (the empty circles) and isolated drops (solid circles). The data from Goldsmith \& Mason (1962) are long drops. Dashed lines represent $\pm 15 \%$ of the exact agreement between theoretical and experimental values.

Fabre \& Line 1992; Brauner \& Ullmann 2004; Picchi et al. 2015) to model slug flow using the correlation by Nicklin, Wilkes \& Davidson (1962). This correlation requires a model for the ascent speed, referred as drift velocity in Nicklin et al. (1962) and identical to the dimensional ascent speed at steady state in our model. To the best of our knowledge, the drift velocity of isolated Taylor drops has only been characterized experimentally (e.g. Wallis 1969; Fabre \& Line 1992), but it has not yet been formulated from first principles.

To fill this gap, we propose to estimate the drift velocity in the non-dissipative regime using (4.3)

$$
\hat{V}_{f}=\left(\frac{\Delta \rho g R^{2}}{\mu_{d}}\right) \frac{(6+2 M) \log 2-M-4}{48+16 M} .
$$

This formula converges to the scaling obtained for the ascent speed of Taylor bubbles in the laminar regime by Wallis (1969) and Brauner \& Ullmann (2004),

$$
\hat{V}_{f}(M \rightarrow \infty)=0.02414 \frac{\Delta \rho g R^{2}}{\mu_{d}}
$$

In the dissipative regime, the drift velocity in slug flow can be estimated by evaluating (2.18) at $\delta_{\infty}=\sqrt{2 \mathcal{R}} / 2$. 
(a)

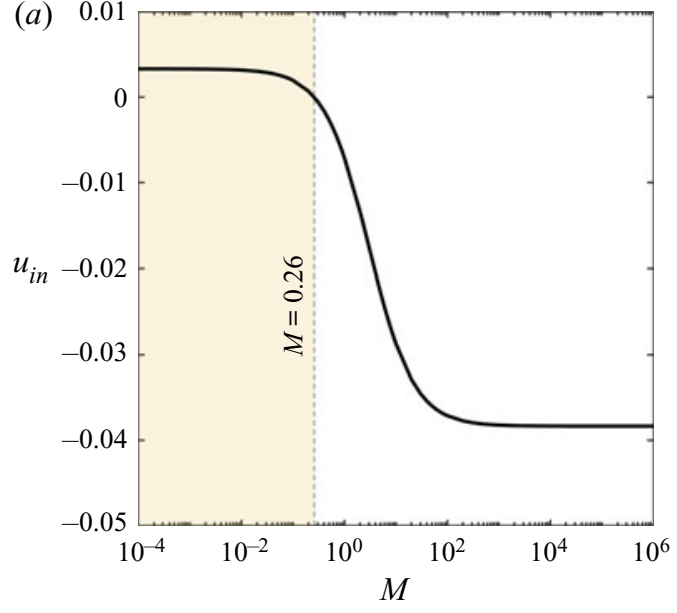

(b)

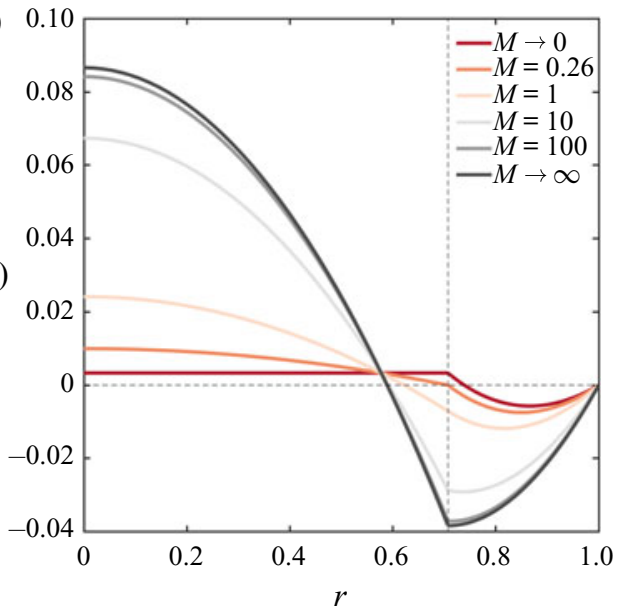

FIGURE 10. (a) Interfacial speed $u_{\text {in }}$ as a function of the viscosity ratio $M$ for the steady state configuration $\delta_{\infty}=\sqrt{2} / 2$ (non-dissipative regime). (b) Velocity profiles of the steady state configuration for different viscosity ratio $M$ in the non-dissipative regime.

\subsection{Backflow and velocity profiles}

The rise of a Taylor drop may lead to backflow in one of two fluids. If the speed at the fluid-fluid interface, $u_{i n}=u_{a}(\delta)=u_{d}(\delta)$, is less than zero, backflow occurs in the ascending fluid. Despite the ascent speed of the ascending fluid being positive, the local velocity assumes a negative value near the interface, highlighting that a portion of the light fluid is dragged downward. The backflow occurs inside the drop as long as $M>0.26$ (see figure $10 a$ ). When $M<0.26$, the ascending fluid drags a portion of the heavy fluid upward and backflow occurs in the descending fluid. The switching point is at $M=0.26$, which corresponds to the case of a static interface $u_{i n}=0$ (see figure $10 a$ ).

Figure $10(b)$ shows the steady-state velocity profiles, $(2.14 a)$ and $(2.14 b)$, for the entire range of viscosity ratios in the non-dissipative regime. It highlights that the velocity profiles converge to the limiting cases of $M \rightarrow \infty$ relatively quickly. In this limit, the velocity profile of the descending fluid resembles that of a free-surface flow. Instead, for $M \rightarrow 0$, the ascending fluid is so viscous that the velocity profile is almost flat. In the dissipative regime, the drop shrinks and the velocity profile changes as depicted in figure $11(b)$. Since $\delta_{\infty}<\sqrt{2} / 2$, the maximal speed at the pipe centre is higher compared with the non-dissipative regime. Also, the magnitude of the interfacial speed increases with the density difference between the fluids, extending the backflow in the descending fluids to higher $M$ compared with the dissipative regime, see figure 11(a).

Our results agree well with the measurements of Goldsmith \& Mason (1962). Figure 12 shows the comparison between the velocity profiles in the axial direction of an isolated Taylor drop measured by Goldsmith \& Mason (1962) for two specific viscosity ratios. The solid curves represent the model predictions in the non-dissipative regime. The model reproduces not only the measured velocity profile, but it also confirms the switching between the two backflow scenarios. For $M=0.91$, backflow occurs in the ascending fluid (see figure 12a), but has shifted into the descending fluid for $M=0.12$ (figure $12 b$ ). These results are consistent with the theoretical switching point at $M=0.26$ and validate the velocity profiles predicted by the model, obtained without any fitting parameter. 

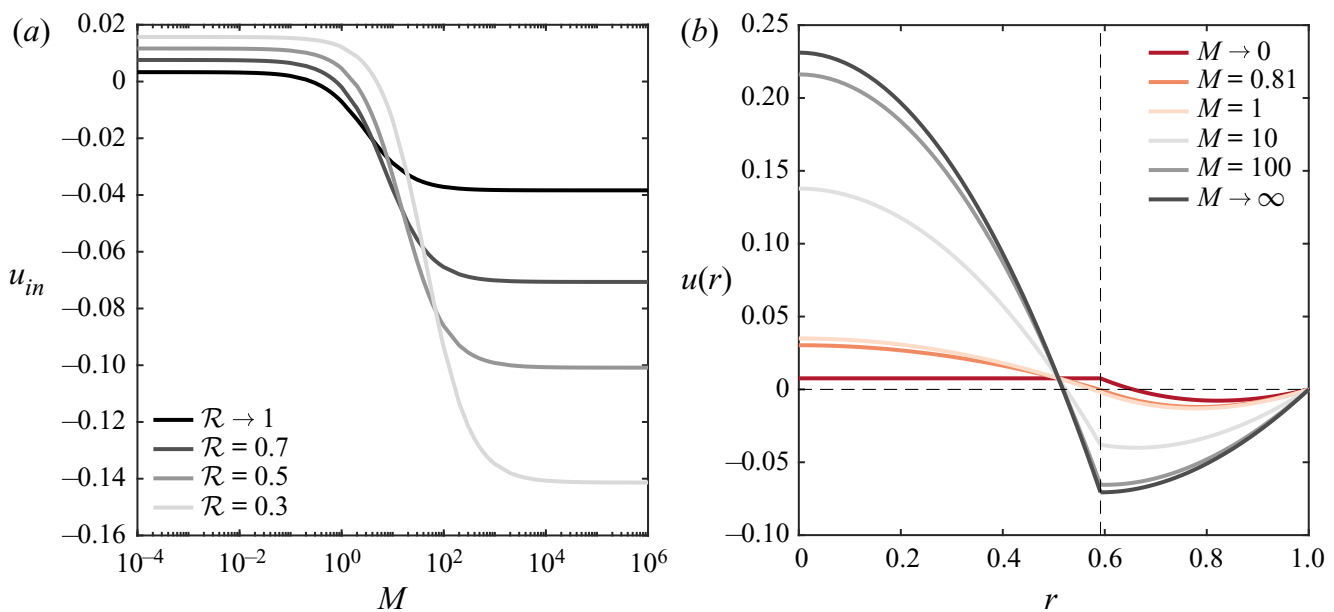

FIGURE 11. (a) Interfacial speed $u_{\text {in }}$ as a function of the viscosity ratio $M$ and the density ratio $\mathcal{R}$ at steady state $\delta_{\infty}=\sqrt{2 \mathcal{R}} / 2$ for the dissipative regime. (b) Velocity profiles at steady state for different viscosity ratio $M$ in the dissipative regime for $\mathcal{R}=0.7$.

(a)

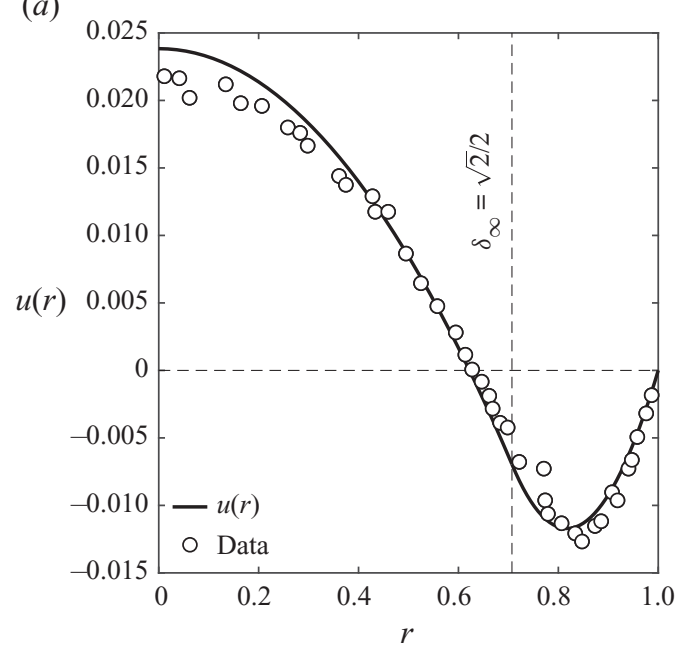

(b)

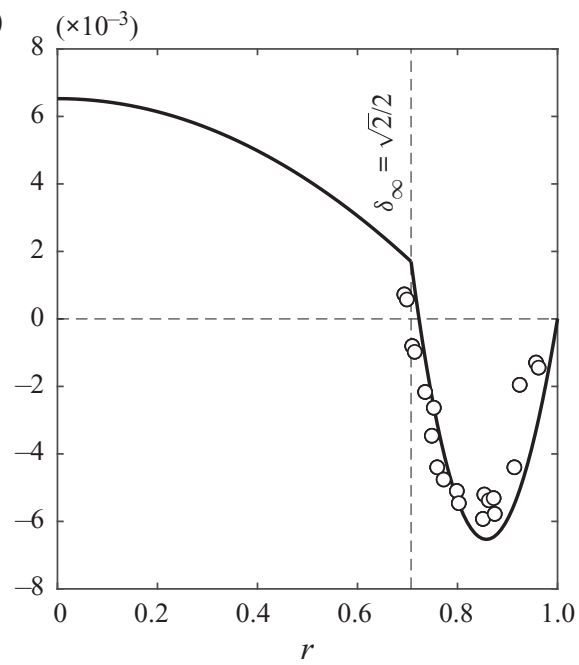

FIGURE 12. Comparison of measured and modelled velocity profiles for the experiments of Goldsmith \& Mason (1962) for $M=0.91(a)$ and $M=0.12(b)$.

Our predicted behaviour in the free-surface limit also agrees well with the numerical simulations of Suckale et al. (2018).

\subsection{Implications for persistently active volcanoes}

In upward and downward inclined flows, multiple configurations may exist for fixed operational conditions (Barnea \& Taitel 1992; Ullmann et al. 2003b; Thibault, Munoz \& Liné 2015; Picchi \& Poesio 2016; Goldstein, Ullmann \& Brauner 2017; Picchi et al. 2017, 2018a; Picchi, Ullmann \& Brauner 2018b). For given fluids flow rates, there exist multiple steady-state solutions that differ in the volume fractions, velocity profiles and the pressure 
drop, but share the same flux. Recent experiments suggest that different configurations may also coexist in the pipe (Ullmann et al. 2003a). Understanding the existence and stability of multiple flow solutions is of particular interest in the volcanological context, because persistently active volcanoes display a wide range of eruptive regimes which are thought to represent different flow configurations in the conduit (e.g. Vergniolle \& Mangan 2000).

Suckale et al. (2018) showed that vertical core-annular flow admits two steady-state configurations with the same flux, a solution with fast flow in a thin core and a solution with relatively slow flow in a thick core. Both solutions appear to be realized in the core-annular flow experiments by Beckett et al. (2011), which involved two large tanks connected by an open pipe. However, only the thick-core solution appears to be physically realized in the lock-exchange set-up of Stevenson \& Blake (1998). The experimental set-up of Stevenson \& Blake (1998) is a closed pipe and the observed flow regime is a Taylor drop instead of core-annular flow.

Here, we show that the presence of a propagating interface (i.e. the Taylor drop) breaks the symmetry between the two solutions derived in Suckale et al. (2018). We find that, for the experiments by Stevenson \& Blake (1998), the energy balance favours a Taylor drop with a constant thickness of $\sqrt{2 \mathcal{R}} / 2$, particularly at high viscosity contrast $M \gg 1$ (see figure $6 c$ ). At smaller viscosity contrast, $M \approx 1$, there is a much wider range of initial configurations that do not lead to the formation of a stable Taylor drop (see figure $6 b$ ). Applied to the volcanic context, this finding suggests that exchange flow in the lava conduit is most prone to instability in the vicinity of the uppermost portion of the conduit, where the viscosity of the two magmatic fluids becomes comparable as the ascending magma loses its volatiles through degassing. Interestingly, mildly eruptive behaviour such as normal eruptions at Stromboli volcano, Italy, tend to originate at relatively shallow depths (e.g. Harris \& Ripepe 2007).

\section{Conclusions}

In this paper, we study the ascent dynamics of a Taylor drop formed from a lock-exchange configuration in a closed vertical pipe. We derive an analytical formulation of the ascent speed in the viscous regime that is consistent with laboratory experiments by combining a lubrication model with the energy balance for the entire system. We show that Taylor drops reach a thickness of $\sqrt{2} / 2$ in steady-state when neglecting dissipation and assume a slightly lower value, $\sqrt{2 \mathcal{R}} / 2$, when dissipation is accounted for. The ascent speed is proportional to the dimensional group $\Delta \rho g R^{2} / \mu_{d}$ and depends on the viscosity ratio in the non-dissipative regime and on both the viscosity ratio and the density ratio in the dissipative regime.

Our study also clarifies that, once the Taylor drop reaches the terminal speed, the system finds an energetic equilibrium. In the non-dissipative regime, the speed at the front equals the speed of the tail and each fluid occupies half of the cross-section of the pipe. In this regime, the ascent dynamics is controlled by the balance of two mechanisms: the loss in the potential energy due to the interpenetration of the lighter fluid into the heavy fluid and the gain in potential energy due to the displacement of the lighter fluid by the heavy fluid. In the dissipative regime, dissipation is a third mechanism that needs to be considered. It causes the drop to shrink and flow faster compared with the non-dissipative regime.

The viscosity ratio controls where backflow occurs. When the viscosity ratio exceeds a threshold value, it occurs inside the drop. Otherwise, backflow switches to the surrounding fluid. Our results have implications for the determination of the drift velocity of a 
Taylor drop in slug flows and our understanding of conduit flow in persistently degassing volcanoes.

\section{Acknowledgements}

D.P. and I.B. were supported by the Department of Energy under the Early Career Award DE-SC0014227. We also thank the anonymous reviewers for their careful reading of our manuscript and their many insightful comments and suggestions.

\section{Declaration of interests}

The authors report no conflict of interest.

\section{Supplementary material}

Supplementary material is available at https://doi.org/10.1017/jfm.2020.596.

\section{Appendix A. Expressions for $g(\delta, t)$ and $f(\delta, t)$}

Here, we report the analytical expressions of the derivatives in $g(\delta, t)$ as follows:

$$
\begin{gathered}
\frac{\partial \mathcal{E}}{\partial t}=\pi \frac{\left(V_{f}-V_{t}\right)}{96}\left[\left(\frac{1}{\mathcal{R}-1}-\delta^{6}-3 \delta^{4}-3 \delta^{2}\right) P^{2}+12(\log \delta)^{2}\left(\delta^{6}+\frac{\delta^{4}}{\mathcal{R}-1}\right)\right. \\
\left.-6 \delta^{2} P \log \delta\left(\delta^{4}-2 \delta^{2}-\frac{1}{\mathcal{R}-1}\right)\right], \\
\frac{\partial \mathcal{P}}{\partial t}=\pi\left[\delta^{2} V_{t}^{2}-\left(1-\delta^{2}\right) V_{f}^{2}\right] t .
\end{gathered}
$$

We obtain the term $g(\delta, t)$ by combining (A 1) and (A 2) with (2.18) and (2.19) as follows:

$$
g(\delta, t)=\frac{1}{R i} \frac{\partial \mathcal{E}}{\partial t}+\frac{\partial \mathcal{P}}{\partial t} .
$$

We compute $f(\delta, t)$ analytically using the chain rule for derivatives of composite functions on $\mathcal{E}=\mathcal{E}\left(\delta, t, V_{f}(\delta), V_{t}(\delta)\right)$ and $\mathcal{P}=\mathcal{P}\left(\delta, t, V_{f}(\delta), V_{t}(\delta)\right)$. Since the analytical expression is quite cumbersome, we do not detail it in the paper but it is available as a Maple file in the supplementary material available at https://doi.org/10.1017/jfm.2020.596.

\section{Appendix B. Energy balance: high $R i$ limit}

In the limit of $R i \rightarrow \infty$, the energy balance (3.24) can be recast as

$$
\frac{\mathrm{d} \delta}{\mathrm{d} t}=-\frac{(1-\mathcal{R}) \Phi+\frac{\partial \mathcal{P}}{\partial t}}{\frac{\partial \mathcal{P}}{\partial \delta}},
$$

where $\partial \mathcal{P} / \partial t$ is given by (A 2) and $\Phi$ is given by (3.18). The term $\partial \mathcal{P} / \partial \delta$ has to be calculated using the chain rule for derivatives of composite functions on $\mathcal{P}=\mathcal{P}\left(\delta, t, V_{f}(\delta), V_{t}(\delta)\right)$. We performed the computation using the software Maple (the 
Maple file is available in the supplementary material of this manuscript). With this, (B 1) becomes

$$
\frac{\mathrm{d} \delta}{\mathrm{d} t}=\frac{a_{1}}{t a_{2}},
$$

where

$$
\begin{aligned}
a_{1}= & \delta\left(\delta^{2}-1\right)\left(\psi-2 \delta^{2}\right)\left[(M-1) \delta^{4}+1\right]\left[\left((M-1) \delta^{4}+1\right) \log \delta\right. \\
& \left.+\left(1-\frac{3 M}{4}\right) \delta^{4}+(M-2) \delta^{2}-\frac{M}{4}+1\right], \\
a_{2}= & {\left[(M-1) \delta^{4}+1\right]^{2}\left(6 \delta^{4}-10 \delta^{2}+3\right) \log \delta } \\
& -\frac{1}{4}\left(\delta^{2}-1\right)\left[\left(10 M^{2}-26 M+16\right) \delta^{10}+\left(-16 M^{2}+44 M-28\right) \delta^{8}\right. \\
& +\left(3 M^{2}+19 M-36\right) \delta^{6}+\left(M^{2}-61 M+108\right) \delta^{4} \\
& \left.+(27 M-76) \delta^{2}-3 M+16\right]
\end{aligned}
$$

and

$$
\begin{array}{ll}
\psi=1 & \text { when } \Phi=0 \\
\psi=\mathcal{R} & \text { when } \Phi \neq 0
\end{array}
$$

\section{REFERENCES}

Alba, K., Taghavi, S. M. \& FrigaArd, I. A. 2014 Miscible heavy-light displacement flows in an inclined two-dimensional channel: a numerical approach. Phys. Fluids 26, 122104.

Amiri, A., Eslami, A., Mollaabbasi, R., Larachi, F. \& Taghavi, S. M. 2019 Removal of a yield stress fluid by a heavier newtonian fluid in a vertical pipe. J. Non-Newtonian Fluid Mech. 268, $81-100$.

Baird, M. H. I., Aravamudan, K., Rao, N. V. R., Chadam, J. \& Peirce, A. P. 1992 Unsteady axial mixing by natural convection in a vertical column. AIChE J. 38 (11), 1825-1834.

BARNEA, D. \& TAITEL, Y. 1992 Structural and interfacial stability of multiple solutions for stratified flow. Intl J. Multiphase Flow 18 (6), 821-830.

Batchelor, G. K. 2000 An Introduction to Fluid Dynamics, Cambridge Mathematical Library. Cambridge University Press.

Beckett, F. M., Mader, H. M., Phillips, J. C., Rust, A. C. \& Witham, F. 2011 An experimental study of low-Reynolds-number exchange flow of two newtonian fluids in a vertical pipe. J. Fluid Mech. 682, 652-670.

Benjamin, T. B. 1968 Gravity currents and related phenomena. J. Fluid Mech. 31 (2), 209-248.

BRAUnER, N. 1998 Liquid-liquid two-phase flows. In Modelling and Experimentation in Two-Phase Flow, pp. 221-279. Springer.

Brauner, N. \& Ullmann, A. 2004 Modelling of gas entrainment from Taylor bubbles. Part 1. Slug flow. Intl J. Multiphase Flow 30 (3), 239-272.

Bretherton, F. P. 1961 The motion of long bubbles in tubes. J. Fluid Mech. 10 (2), 166-188.

BROWN, R. A. S. 1965 The mechanics of large gas bubbles in tubes: I. Bubble velocities in stagnant liquids. Can. J. Chem. Engng 43 (5), 217-223.

DAVIES, R. M. \& TAYLOR, G. I. 1950 The mechanics of large bubbles rising through extended liquids and through liquids in tubes. Proc. R. Soc. Lond. A 200 (1062), 375-390.

Direito, F. J. N., CAmpos, J. B. L. M. \& Miranda, J. M. 2016 Rising of a single Taylor drop in a stagnant liquid-2D laminar flow and axisymmetry limits. Phys. Fluids 28, 057101. 
Dumitrescu, D. T. 1943 Stromung an einer luftblase im senkrechten rohr. Z. Angew. Math. Mech. 23 (3), $139-149$.

Dussan, V. E. B. 1975 Hydrodynamic stability and instability of fluid systems with interfaces. Arch. Rat. Mech. Anal. 57 (4), 363-379.

ETRATI, A. \& FRIGAARD, I. A. 2018 A two-layer model for buoyant inertial displacement flows in inclined pipes. Phys. Fluids 30, 022107.

Fabre, J. \& Line, A. 1992 Modeling of two-phase slug flow. Annu. Rev. Fluid Mech. 24 (1), 21-46.

Fowler, A. C. \& Robinson, M. 2018 Counter-current convection in a volcanic conduit. J. Volcanol. Geotherm. Res. 356, 141-162.

Francis, P., Oppenheimer, C. \& Stevenson, D. 1993 Endogenous growth of persistently active volcanoes. Nature 366 (6455), 554.

FRIgAARD, I. A. \& SCHERZER, O. 1998 Uniaxial exchange flows of two Bingham fluids in a cylindrical duct. IMA J. Appl. Maths 61 (3), 237-266.

Giordano, D., Russell, J. K. \& Dingwell, D. B. 2008 Viscosity of magmatic liquids: a model. Earth Planet. Sci. Lett. 271 (1-4), 123-134.

Goldsmith, H. L. \& MASON, S. G. 1962 The movement of single large bubbles in closed vertical tubes. J. Fluid Mech. 14 (1), 42-58.

Goldstein, A., Ullmann, A. \& BRAUner, N. 2017 Exact solutions of core-annular laminar inclined flows. Intl J. Multiphase Flow 93, 178-204.

HARRIS, A. \& RIPEPE, M. 2007 Synergy of multiple geophysical approaches to unravel explosive eruption conduit and source dynamics-a case study from stromboli. Geochemistry 67 (1), 1-35.

Hasnain, A. \& Alba, K. 2017 Buoyant displacement flow of immiscible fluids in inclined ducts: a theoretical approach. Phys. Fluids 29, 052102.

Hasnain, A., Segura, E. \& Alba, K. 2017 Buoyant displacement flow of immiscible fluids in inclined pipes. J. Fluid Mech. 824, 661-687.

Hayashi, K., Kurimoto, R. \& Tomiyama, A. 2011 Terminal velocity of a Taylor drop in a vertical pipe. Intl J. Multiphase Flow 37 (3), 241-251.

Joseph, D. D., NGUYen, K. \& BeAVERs, G. S. 1984 Non-uniqueness and stability of the configuration of flow of immiscible fluids with different viscosities. J. Fluid Mech. 141, 319-345.

Joseph, D. D. \& Renardy, Y. Y. 1992 Fundamentals of two-fluid dynamics. In Fundamentals of Two-Fluid Dynamics. Springer.

Kazahaya, K., Shinohara, H. \& Saito, G. 1994 Excessive degassing of Izu-Oshima volcano: magma convection in a conduit. Bull. Volcanol. 56 (3), 207-216.

Kerswell, R. R. 2011 Exchange flow of two immiscible fluids and the principle of maximum flux. J. Fluid Mech. 682, 132-159.

Kurimoto, R., Hayashi, K. \& Tomiyama, A. 2013 Terminal velocities of clean and fully-contaminated drops in vertical pipes. Intl J. Multiphase Flow 49, 8-23.

Llewellin, E. W., Bello, E. D., Taddeucci, J., Scarlato, P. \& Lane, S. J. 2012 The thickness of the falling film of liquid around a Taylor bubble. Proc. R. Soc. Lond. A 468 (2140), 1041-1064.

MirzaeiAn, N. \& ALBA, K. 2018 Monodisperse particle-laden exchange flows in a vertical duct. J. Fluid Mech. 847, 134-160.

Nicklin, D. J., Wilkes, J. O. \& Davidson, J. F. 1962 Two phase flow in vertical tubes. Trans. Inst. Chem. Engrs 40, 61-68.

Oladosu, O., Hasnain, A., Brown, P., FrigaArd, I. \& Alba, K. 2019 Density-stable displacement flow of immiscible fluids in inclined pipes. Phys. Rev. Fluids 4 (4), 044007.

PiCCHI, D., BARMAK, I., UlLmANN, A. \& BRAUNER, N. 2018a Stability of stratified two-phase channel flows of newtonian/non-newtonian shear-thinning fluids. Intl J. Multiphase Flow 99, 111-131.

Picchi, D., Manerba, Y., Correra, S., Margarone, M. \& Poesio, P. 2015 Gas/shear-thinning liquid flows through pipes: modeling and experiments. Intl J. Multiphase Flow 73, 217-226.

PICCHI, D. \& PoEsio, P. 2016 Stability of multiple solutions in inclined gas/shear-thinning fluid stratified pipe flow. Intl J. Multiphase Flow 84, 176-187.

Picchi, D., Poesio, P., Ullmann, A. \& Brauner, N. 2017 Characteristics of stratified flows of newtonian/non-newtonian shear-thinning fluids. Intl J. Multiphase Flow 97, 109-133. 
Picchi, D., Ullmann, A. \& Brauner, N. $2018 b$ Modeling of core-annular and plug flows of newtonian/non-newtonian shear-thinning fluids in pipes and capillary tubes. Intl J. Multiphase Flow 103, 43-60.

REINELT, D. A. 1987 The rate at which a long bubble rises in a vertical tube. J. Fluid Mech. 175, 557-565.

SAHU, K. C. \& VANKA, S. P. 2011 A multiphase lattice Boltzmann study of buoyancy-induced mixing in a tilted channel. Comput. Fluids 50 (1), 199-215.

Shin, J. O., DAlziel, S. B. \& Linden, P. F. 2004 Gravity currents produced by lock exchange. J. Fluid Mech. 521, 1-34.

Shukla, I., Kofman, N., Balestra, G., Zhu, L. \& Gallaire, F. 2019 Film thickness distribution in gravity-driven pancake-shaped droplets rising in a Hele-Shaw cell. J. Fluid Mech. 874, 1021-1040.

Stevenson, D. S. \& Blake, S. 1998 Modelling the dynamics and thermodynamics of volcanic degassing. Bull. Volcanol. 60 (4), 307-317.

Suckale, J., Qin, Z., Picchi, D., Keller, T. \& Battiato, I. 2018 Bistability of buoyancy-driven exchange flows in vertical tubes. J. Fluid Mech. 850, 525-550.

Thibault, D., Munoz, J.-M. \& Liné, A. 2015 Multiple holdup solutions in laminar stratified flow in inclined channels. Intl J. Multiphase Flow 73, 275-288.

Ullmann, A., ZAmiR, M., GAT, S. \& BRAUner, N. 2003a Multi-holdups in co-current stratified flow in inclined tubes. Intl J. Multiphase Flow 29 (10), 1565-1581.

Ullmann, A., ZAmiR, M., Ludmer, Z. \& BRAUner, N. 2003b Stratified laminar countercurrent flow of two liquid phases in inclined tubes. Intl J. Multiphase Flow 29 (10), 1583-1604.

Vergniolle, S. \& Mangan, M. 2000 Hawaiian and Strombolian eruptions. In Encyclopedia of Volcanoes (ed. H. Sigurdsson, B. Houghton, S. McNutt, H. Rymer \& J. Stix), pp. 447-461. Elsevier.

Viana, F., Pardo, R., Yanez, R., Trallero, J. L. \& Joseph, D. D. 2003 Universal correlation for the rise velocity of long gas bubbles in round pipes. J. Fluid Mech. 494, 379-398.

Wallis, G. B. 1969 One-Dimensional Two-Phase Flow. McGraw-Hill.

White, E. T. \& BEARDMORE, R. H. 1962 The velocity of rise of single cylindrical air bubbles through liquids contained in vertical tubes. Chem. Engng Sci. 17 (5), 351-361.

ZUKOSKI, E. E. 1966 Influence of viscosity, surface tension, and inclination angle on motion of long bubbles in closed tubes. J. Fluid Mech. 25 (4), 821-837. 